\title{
Kalirin-7 Is an Essential Component of both Shaft and Spine Excitatory Synapses in Hippocampal Interneurons
}

\author{
Xin-Ming Ma, Yanping Wang, Francesco Ferraro, Richard E. Mains, and Betty A. Eipper \\ Department of Neuroscience, University of Connecticut Health Center, Farmington, Connecticut 06030
}

\begin{abstract}
Kalirin, a multifunctional Rho GDP/GTP exchange factor, plays a vital role in cytoskeletal organization, affecting process initiation and outgrowth in neurons. Through alternative splicing, the Kalirin gene generates multiple functionally distinct proteins. Kalirin-7 (Kal7) is the most prevalent isoform in the adult rat hippocampus; it terminates with a postsynaptic density-95 (PSD-95)/Discs large/zona occludens-1 (PDZ) binding motif, is localized to the postsynaptic density, interacts with PSD-95, and causes the formation of dendritic spines when overexpressed in pyramidal neurons. Levels of Kal7 are low in the dendrites of hippocampal aspiny interneurons. In these interneurons, Kal7 is localized to the postsynaptic side of excitatory synapses onto dendritic shafts, overlapping clusters of PSD-95 and NMDA receptor subunit NR1. Selectively decreasing levels of Kal7 decreases the density of PSD-95-positive, bassoon-positive clusters along the dendritic shaft of hippocampal interneurons. Overexpression of Kal7 increases dendritic branching, inducing formation of spine-like structures along the dendrites and on the soma of normally aspiny hippocampal interneurons. Essentially all of the spine-like structures formed in response to Kal7 are apposed to vesicular glutamate transporter 1-positive, bassoon-positive presynaptic endings; GAD-positive, vesicular GABA transporter-positive inhibitory endings are unaffected. Almost every Kal7-positive dendritic cluster contains PSD-95 along with NMDA (NR1) and AMPA (GluR1 and GluR2) receptor subunits. Kal7-induced formation of spine-like structures requires its PDZ binding motif, and interruption of interactions between the PDZ binding motif and its interactors decreases Kal7-induced formation of spine-like structures. Kal7 thus joins Shank3 and GluR2 as molecules with a level of expression at excitatory synapses that titrates the number of dendritic spines.
\end{abstract}

Key words: dendrite; dendritic spine; synaptogenesis; Rho GEF; PSD-95; GABA; dendritic growth

\section{Introduction}

A delicate balance between synaptic excitation and inhibition is critical for maintaining normal circuits in the CNS, and interneurons play an essential role in regulating local circuit excitability (Maccaferri and Lacaille, 2003). Most hippocampal interneurons release GABA as their primary neurotransmitter (Freund and Buzsaki, 1996). Disruption of GABAergic interneuron development may play a role in epilepsy, bipolar disorder, schizophrenia, autism, and Alzheimer's disease (Benes and Berretta, 2001; Powell et al., 2003; Ramos et al., 2006).

Based on their morphological, biochemical, and electrophysiological properties, hippocampal interneurons are diverse, allowing them to play a wide range of roles in information processing (Freund and Buzsaki, 1996; Parra et al., 1998; McBain and Fisahn, 2001; Maccaferri and Lacaille, 2003). Neurochemical markers for interneurons include calcium binding proteins and neuropeptides (Sloviter and Nilaver, 1987; Gulyas et al., 1991; Baraban and Tallent, 2004). Hippocampal interneurons are generally free of spines, and both excitatory and inhibitory inputs synapse onto the dendritic shaft (Benson et al., 1994; Gulyas et al., 1999).

Received 0ct. 31, 2006; accepted Nov. 29, 2007.

This work was supported by National Institutes of Health Grant DA-015464 (B.A.E.). We thank Darlene D'Amato for her laboratory support and Drs. Zhao-wen Wang and Eric Levine for their comments on this manuscript.

Correspondence should be addressed to Dr. Xin-Ming Ma, Department of Neuroscience, University of Connecticut Health Center, 263 Farmington Avenue, MC-3401, Farmington, CT 06030-3401. E-mail: Ma@nso.uchc.edu. DOI:10.1523/JNEUROSCI.5283-07.2008

Copyright $\odot 2008$ Society for Neuroscience $\quad$ 0270-6474/08/280711-14\$15.00/0
Hippocampal interneurons express several $\mathrm{GABA}_{\mathrm{A}}$ receptor subunits, and their dendritic shafts receive inhibitory inputs from GABAergic endings (Mody and Pearce, 2004). Formation of spiny versus aspiny excitatory synapses is cell-type specific and developmentally regulated (Rao et al., 1998; Anderson et al., 2004). Like excitatory synapses onto spines, aspiny excitatory glutamatergic postsynaptic endings contain postsynaptic density-95 (PSD-95) and glutamate receptors, GABAergic inhibitory postsynaptic endings do not (McBain et al., 1999; Zhang et al., 1999; Craig et al., 2006). Expression of the extracellular domain of the AMPA receptor subunit GluR2 causes spine formation in hippocampal interneurons (Passafaro et al., 2003). Synapse-specific calcium compartmentalization can be achieved at both spiny and aspiny excitatory synapses.

Small GTPases of the Rho subfamily and their GDP/GTP exchange factors (GEFs) play vital roles in the function of dendritic spines in hippocampal pyramidal neurons and cortical neurons (Nakayama et al., 2000; Tashiro et al., 2000; Ryan et al., 2005; Tolias et al., 2005). Kalirin, a multifunctional Rho GEF, is expressed in both hippocampal pyramidal neurons and interneurons (Ma et al., 2001). Antisense-mediated reductions in endogenous Kalirin expression (all isoforms) in hippocampal pyramidal neurons caused simplification of the dendritic tree and a reduction in spine density (Ma et al., 2003). Kal7, the major splice variant in the adult rat hippocampus, terminates with a PSD-95/Discs large/zona occludens-1 (PDZ)-binding motif, interacts with PSD-95, and localizes to the postsynaptic density 
(Penzes et al., 2001b; Ma et al., 2003) (see Fig. 1A). Overexpression of Kal7 in cortical neurons causes an increase in spine density (Penzes et al., 2001b). Because hippocampal interneurons primarily lack dendrite spines (Benson et al., 1994; Gulyas et al., 1999) but do express Kal7, our aim was to identify the roles of Kal7 in this neuronal cell type. We show that overexpression of Kal7 in hippocampal interneurons causes formation of spine synapses and that endogenous Kal7 is essential for maintenance of excitatory synapses onto dendritic shafts. The formation of spine synapses in interneurons induced by Kal7 requires its $\mathrm{PDZ}$ binding motif, presumably via its binding of interactors. A better understanding of the roles of Kalirin in interneurons will contribute to understanding learning and memory and to developing strategies for the treatment of neurological and psychiatric disorders (Wang et al., 2006).

\section{Materials and Methods \\ Animals}

Timed pregnant adult female Sprague Dawley rats from Charles River Laboratories were housed one per cage with a 14/10 h light/dark cycle with food and water available ad libitum. All experiments were conducted in accordance with the guidelines established by the University of Connecticut Health Center Animal Care and Use Committee. For preparations of organotypic slices and dissociated cells, rat pups were killed at postnatal day 9 (P9) or P1, respectively. For immunohistochemistry, adult male Sprague Dawley rats were anesthetized with ketamine and perfused transcardially with saline followed by $4 \%$ paraformaldehyde in PBS.

\section{Antibodies}

The following rabbit antibodies were used: affinity-purified Kal7 (JH2959, 1:200), GAD65/67 (1:2000; Sigma, St. Louis, MO), GluR1 (1:500; Millipore, Billerica, MA), vesicular GABA transporter (VGAT) (1:1000; Synaptic Systems, Goettingen, Germany), green fluorescent protein (GFP) (for Western blot analysis; Abcam, Cambridge, UK), microtubuleassociated protein 2 (MAP2) (1:500; Chemicon, Temecula, CA), neuropeptide Y (NPY) (JH4; 1:1000), peptidylglycine alpha-amidating monooxygenase (PAM) (JH629; 1:500). The following mouse monoclonal antibodies were used: PSD-95 (clone28/43, 1:300; gift from Dr. Matt Rasband, University of Connecticut Health Center), Bassoon (1:200; Stressgen, Ann Arbor, MI), NMDA receptor subunit NR1 (1:300; PharMingen, San Diego, CA), GluR2 (1:500; Chemicon), GAD65 (GAD6, 1:200; Developmental Studies Hybridoma Bank, University of Iowa, Iowa City, IA), parvalbumin (PV) (1:2000; Swant, Bellinzona, Switzerland), MAP2 (1:500; Sigma), $\beta$ III-tubulin (TUJ1; $1: 500$; Covance, Princeton, NJ), $\mathrm{GABA}_{\mathrm{A}}$ receptor ( $\beta$-chain, 1:200; Chemicon), GFP (1: 200; Chemicon), Myc (9E10; 1:20), Kal7 (1:10; clone 20-D8). Guinea pig antibody to vesicular glutamate transporter 1 (VGLUT1) (1:3000; Chemicon) and rat antibody to GFP (1:1000; Nacalai Tesque, Japan) were used where indicated.

\section{DNA constructs}

pEAK-Kal7GFP constructs. Enhanced GFP (EGFP) and monomeric (A206K)EGFP were appended to the $\mathrm{N}$ terminus or inserted into sites
Spectrin GEF-1 GEF-2 IgFn Kinase

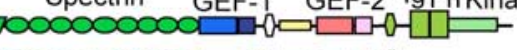

$0 \square+0$

PDZ-binding motif

- Kal7 probe \& antibody
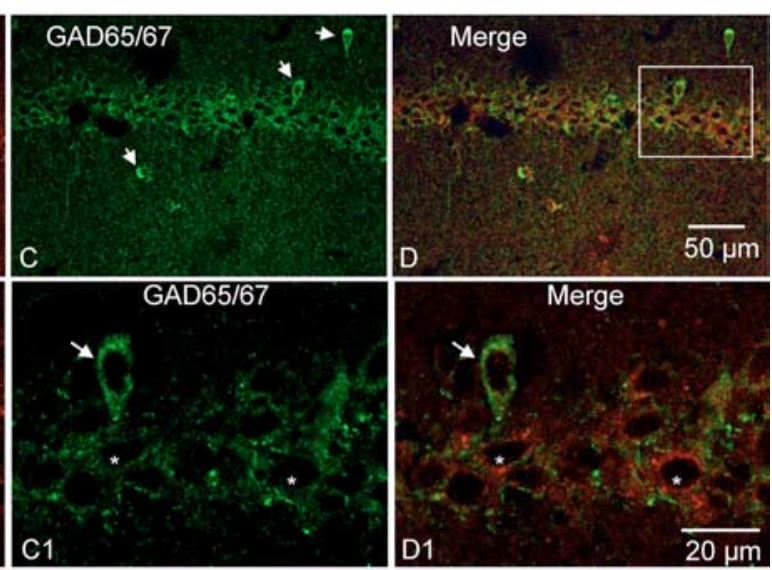

C1
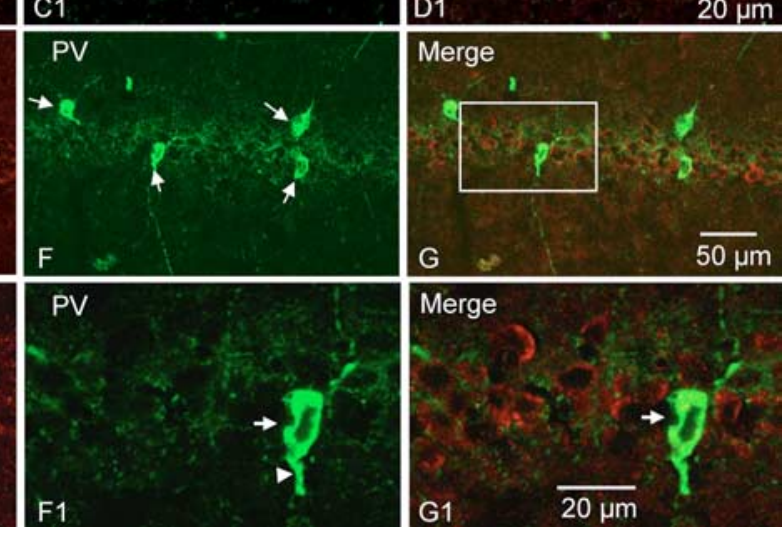

Figure 1. Kal7 is expressed in hippocampal interneurons. $\boldsymbol{A}$, The Kalirin gene generates at least 10 transcripts encoding functionally distinct proteins. The largest isoform, Kal12, has multiple spectrin-like repeats, two Rho GEF domains, and a kinase and GAD65/67 (C) identified Kal7 in interneurons (defined as GAD-positive) in the CA1 region of the adult rat hippocampus. Arrows ( Kal7 in PV-positive interneurons (arrows) in the CA1 region of the adult rat hippocampus. E1-G1 are high-powe images from boxed areas of images $\boldsymbol{E}-\boldsymbol{G}$, respectively. The dendrite (arrowhead) extending from the soma of the PV-positive neuron does not contain a high level of Kal7.

within spectrin repeats 5 and 9 of HisMycKal7 in the pEAK10 vector (Edge Biosystems, Gaithersburg, MD). Each of the EGFP-tagged variants of Kal7 produced massive lamellipodia and activated Rac when expressed in fibroblasts, but N-terminally EGFP-tagged Kal7 formed large fluorescent aggregates. Kal7 with EGFP or monomeric EGFP inserted into spectrin repeat 9 did not form large aggregates when expressed in hippocampal neurons. The GEF activity of GFP-tagged Kal7 was verified using cell-based Rac activation assays as described previously (May et al., 2002; Schiller et al., 2005). pEAK vectors encoding HisMyc-Kal-GEF1 and HisMycKal7 were described previously (May et al., 2002). pCW-PSD-95 was a generous gift from Dr. Morgan Sheng (Harvard University, Cambridge, MA). pSCEP-Kalirin-7- $\Delta$ CT was generated by replacement of the NsiINotI fragment of pSCEP-Kalirin-7 with a fragment truncated after nt 4728 of Kalirin-7, thus encoding a protein lacking the C-terminal 60 amino acids. For pCMS-Kal7-antisense, the dual promoter pCMS vector (BD Biosciences, Franklin Lakes, NJ; Clontech, Cambridge, UK) puts EGFP expression under control of the SV40 promoter and expression of the gene of interest under control of the CMV promoter. The $60 \mathrm{nt}$ of coding region unique to Kal7 mRNA plus the following $845 \mathrm{nt}$ ( $5^{\prime}$-GGC ACC CTT GTT to TCC AAT TTT TCA T-3') of $3^{\prime}$-untranslated region 


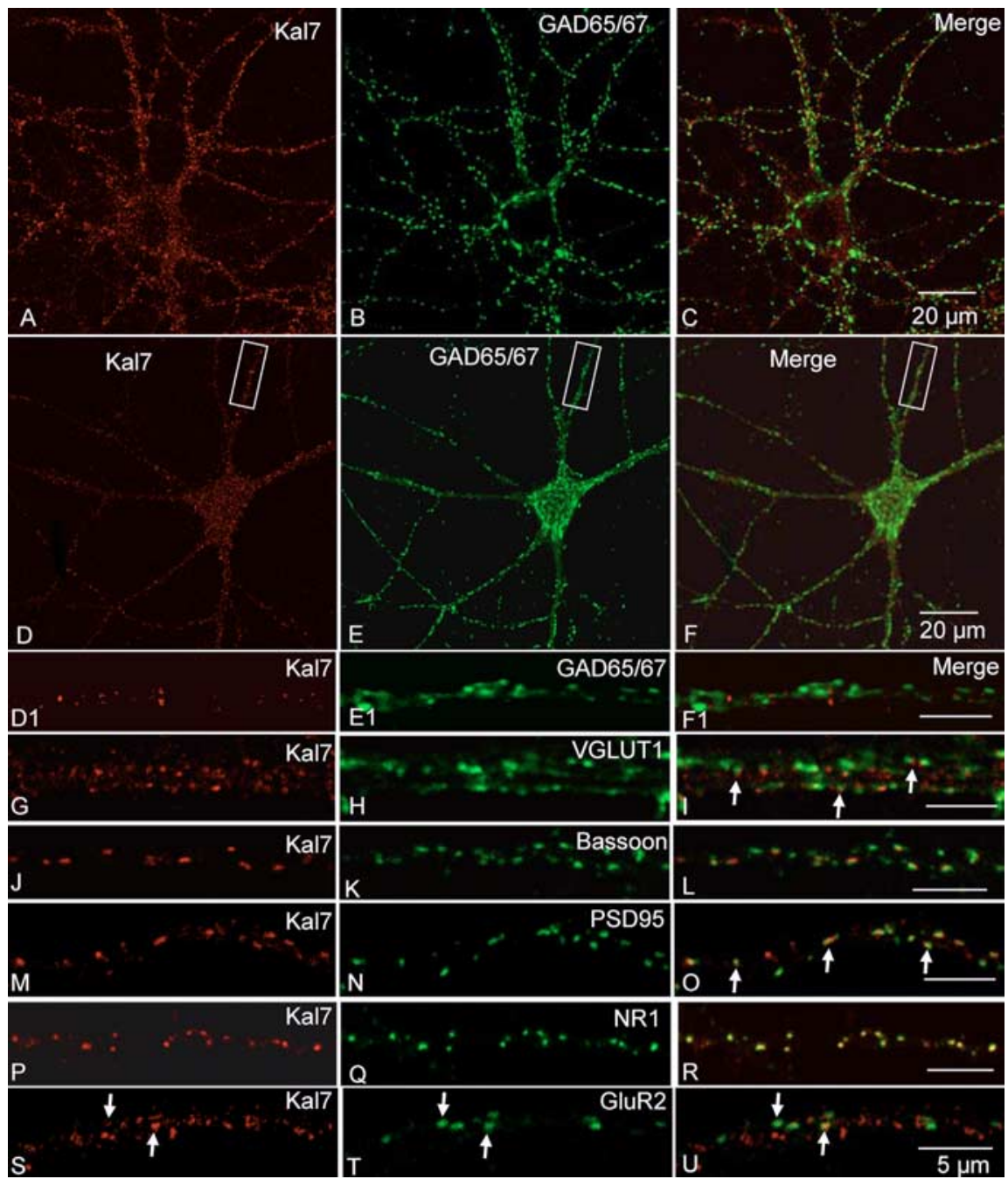

Figure 2. Kal7 is localized at excitatory synapses of hippocampal interneurons. $\boldsymbol{A}-\boldsymbol{F}$, Double immunostaining of dissociated hippocampal neurons (21 DIV) reveals Kal7 $(\boldsymbol{A}, \boldsymbol{D})$ in the soma and dendrites of GAD65/67-negative $(\boldsymbol{B})$ and GAD65/67-positive $(\boldsymbol{E})$ cells. GAD65/67-positive terminals surround the GAD65/67-negative soma (B). D1-F1, Enlargement of dendrite from the boxed area of $\boldsymbol{D}-\boldsymbol{F}$ shows that Kal7 (D1) is not colocalized with GAD-positive $(\boldsymbol{E} 1)$ inhibitory presynaptic terminals $(\boldsymbol{F 1}$, merge). $\mathbf{G}-\boldsymbol{U}$, Dendrites of hippocampal interneurons were stained simultaneously for Kal7 $(\boldsymbol{G}, \boldsymbol{J}, \boldsymbol{M}, \boldsymbol{P}, \boldsymbol{S})$ and VGLUT1 (H, marker for excitatory presynaptic terminals), Bassoon ( $\boldsymbol{K}$, presynaptic marker), PSD-95 ( $\boldsymbol{N}$, postsynaptic density protein), NMDA receptor ( $\boldsymbol{Q}$, NR1), or AMPA receptor subunit GluR2 $(\boldsymbol{T})$. Merged images indicate that Kal7 is localized on the postsynaptic side of excitatory synapses. Interneurons were identified by GAD65/67 staining (data not shown). Arrows show the relationship of two synaptic proteins. I, Kal7 clusters were always aligned with VGLUT1 clusters. 0, Kal7 clusters were always positive for PSD-95. U, Kal7 clusters were not always positive for CluR2. were cloned into pCMS-EGFP in the antisense orientation. The cDNA was amplified by PCR from rat brain mRNA, and the DNA sequence was verified against the rat genome (http://genome.ucsc.edu/). pDsRedMonomer-N1 (Clontech) expresses DsRed under control of the CMV promoter. For pSIREN-Kal7 short hairpin RNA (shRNA) constructs and pSIREN-PAM shRNA constructs, the RNAi-Ready-pSiren-DNRDsRed-Express vector (Clontech) places shRNA expression under control of the human U6 promoter and DsRed expression under control of the CMV promoter. Sequences for shRNAs were selected using the rules at http://bioinfo.clontech.com/rnaidesigner/frontpage.jsp. pSIRENKal7 shRNA encodes a Kal7 shRNA located $394 \mathrm{nt}$ after the stop codon in the unique 3 '-untranslated region (UTR) region of rat Kal7 mRNA (374 nt after the stop codon in mouse) (5'-AAAGTCTGCAACTCAAGTA$\left.3^{\prime}\right)$. pSIREN-PAM shRNA encodes a PAM shRNA within the PHM domain (5'-AACCTGTACAGATAAAGCC-3'). All DNA constructs were verified by sequencing.
Primary cultures of rat hippocampal neurons and transfection

Dissociated hippocampal cultures were prepared from P1 Sprague Dawley rats as described previously (Ma et al., 2003). Briefly, the hippocampi were digested with $0.25 \%$ trypsin for $25 \mathrm{~min}$ at $37^{\circ} \mathrm{C}$. Dissociated cells were plated in Neurobasal A medium containing 7\% heatinactivated horse serum, $1 \times \mathrm{B} 27$ supplement, and $0.5 \mathrm{~mm}$ glutamine and maintained at $37^{\circ} \mathrm{C}$ in $5 \% \mathrm{CO}_{2}$. Three hours later, plating medium was replaced with fresh medium containing only $3 \%$ horse serum (heat inactivated). Three days after plating, the culture medium was exchanged with maintenance medium (Neurobasal A medium containing 2\% B27 supplement, $0.5 \mathrm{~mm}$ glutamine, $25 \mathrm{U} / \mathrm{ml}$ penicillin, 25 $\mu \mathrm{g} / \mathrm{ml}$ streptomycin). Thereafter, $50 \%$ of the medium was replaced twice a week for up to 3 weeks. Freshly dissociated P1 neurons were nucleofected (Amaxa, Germany) using the rat neuron kit. After nucleofection, neurons were plated and allowed to recover in DMEM for $3 \mathrm{~h}$; nucleofected neurons were kept in maintenance medium up to 3 weeks.

\section{Treatment of hippocampal cultures with} cell permeant peptide

To disrupt binding of the Kal7 PDZ binding motif to its interactors, we synthesized a peptide comprising the final eight residues of Kal7 (GDPFSTYV; Kal7CT) preceded by seven arginine residues (R7-Kal7CT). In the control peptide, the C-terminal Val was replaced with Asp (GDPFSTYD) (mutant R7 Kal7CT). To determine whether the peptides entered neurons, dissociated hippocampal neurons [18 d in vitro (DIV)] were incubated with the R7-Kal7CT $(1,5,10,20$ $\mu \mathrm{M}$, peptides dissolved in medium) for $45 \mathrm{~min}$; neurons were then washed with medium and returned to the incubator with half the original medium. Control neurons were treated with mutant R7-Kal7CT $(1,5,10$, and $20 \mu \mathrm{M})$ or vehicle at the same time under the same conditions. Neurons were fixed with $4 \%$ paraformaldehyde $1 \mathrm{~h}, 12 \mathrm{~h}, 24 \mathrm{~h}$, or $3 \mathrm{~d}$ after removing peptide and double stained with a polyclonal antibody to Kal7 (which detects both the peptide and endogenous Kal7) and a monoclonal antibody to Kal7 (which detects endogenous Kal7, but not R7-Kal7CT). Based on signal-tonoise ratio, the optimal concentration of peptide is $10 \mu \mathrm{M}$. To determine whether R7Kal7CT can alter Kal7-induced spine formation, dissociated hippocampal neurons were transfected with vector encoding Kal7GFP at DIV1. The R7-Kal7CT peptide $(10 \mu \mathrm{M})$ was added to the cultures at 7 DIV and 13 DIV. Control neurons received mutant R7-Kal7CT $(10 \mu \mathrm{M})$ or vehicle at the same times. Cells were fixed with $4 \%$ paraformaldehyde and triple stained at 18 DIV with antibodies to GFP, MAP2, and GAD65.

\section{Preparation of rat hippocampal organotypic cultures}

Hippocampal slices were prepared from P9 Sprague Dawley rats as described previously (Ma et al., 2003). Briefly, hippocampi were dissected into ice-cold, sterile Gey's balanced salt solution (Sigma) containing $0.5 \%$ glucose. Medial hippocampi were sliced transversely $(400 \mu \mathrm{m})$ using a slice chopper. Slices were kept in ice-cold Gey's balanced salt solution and then placed onto $30 \mathrm{~mm}$ Millicell CM membrane inserts in Petri dishes filled with $1.1 \mathrm{ml}$ of culture medium containing $0.5 \times$ basal Eagle's medium, $0.25 \times$ HBSS, $0.25 \times$ horse serum (defined, heat- 
inactivated), $25 \mathrm{U} / \mathrm{ml}$ penicillin, $25 \mu \mathrm{g} / \mathrm{ml}$ streptomycin, and $1 \mathrm{~mm}$ L-glutamine (Invitrogen). Slices were kept under $5 \% \mathrm{CO}_{2}$ at $37^{\circ} \mathrm{C}$, with media changes at 1 DIV and every $3 \mathrm{~d}$ thereafter.

\section{Immunohistochemistry (brain sections and slices)}

Coronal sections $(12 \mu \mathrm{m})$ were cut and mounted on gelatin-coated slides. Antibody specificity was evaluated by replacement of antibody with preimmune serum and preincubation of antibody with its antigen $(10 \mu \mathrm{g} / \mu \mathrm{l})$ as described previously (Ma et al., 2003); staining was eliminated in both controls. Sections were blocked in PBS containing 1\% BSA $10 \%$ normal goat serum $/ 0.25 \%$ Triton X-100, $\mathrm{pH} 7.4$, for $1 \mathrm{~h}$ at room temperature. Sections were stained subsequently with polyclonal Kal7 and GAD65/67 antibodies at $4^{\circ} \mathrm{C}$ overnight. Sections were stained simultaneously with polyclonal Kal7 and monoclonal parvalbumin antibodies. Primary antibodies were visualized with Cy3-labeled donkey anti-rabbit IgG (The Jackson Laboratory, Bar Harbor, ME) and FITClabeled goat anti-mouse IgG (The Jackson Laboratory). Images were taken with a Zeiss LSM510 confocal microscope. Replacement of the Kal7 antibody with preimmune serum or preincubation of the antibody with its antigen $(10 \mu \mathrm{g} / \mathrm{ml})$ completely eliminated staining (Ma et al., 2003).

Hippocampal slices were fixed for immunostaining in $4 \%$ paraformaldehyde in PBS as described previously (Ma et al., 2003). Slices were stained simultaneously with polyclonal Kal7 and monoclonal GFP antibodies and visualized as described above. For measurement of Kal7 staining intensity in Kal7GFP neurons and neighboring nontransfected neurons, images were taken under identical conditions and NIH Image J was used to analyze fluorescence intensity (Ma et al., 2003). At least 20 neurons were analyzed for each group.

\section{Immunocytochemistry}

\section{(dissociated neurons)}

Neurons were generally fixed for $20 \mathrm{~min}$ in $4 \%$ paraformaldehyde in PBS. After blocking in PBS containing $1 \% \mathrm{BSA} / 5 \%$ normal goat or donkey (or both) serum $/ 0.20 \%$ Triton X-100, $\mathrm{pH} 7.4$, for $1 \mathrm{~h}$ at room temperature, cells were double or triple stained with appropriate primary antibodies overnight at $4^{\circ} \mathrm{C}$. For visualization of NR1, VGLUT1, GluR1, GluR2, VGAT, $\mathrm{GABA}_{\mathrm{A}}$ receptor, and $\mathrm{Kal}$, cells were fixed with methanol for $12 \mathrm{~min}$ at $-20^{\circ} \mathrm{C}$, except where indicated. Primary antibodies were visualized with appropriate secondary antibodies: Cy3 donkey anti-rabbit IgG (The Jackson Laboratory), Cy3 donkey anti-mouse IgG (The Jackson Laboratory), Cy3 donkey anti-guinea pig IgG (The Jackson Laboratory), FITC goat anti-mouse IgG (The Jackson Laboratory), FITC goat anti-rat IgG (The Jackson Laboratory), Alexa Fluor 633-goat anti-rabbit IgG (Invitrogen), Alexa Fluor 633 goat anti-mouse IgG (Invitrogen). Coverslips were mounted with Prolong Gold antifade reagent (Invitrogen).

\section{Biolistic transfection}

Plasmid DNA was precipitated onto $1.0 \mu$ m gold microcarrier particles at a concentration of $1 \mu \mathrm{g}$ plasmid/mg gold as described previously (Ma et al., 2003). At 2 DIV, slices were transfected with plasmid-coated gold
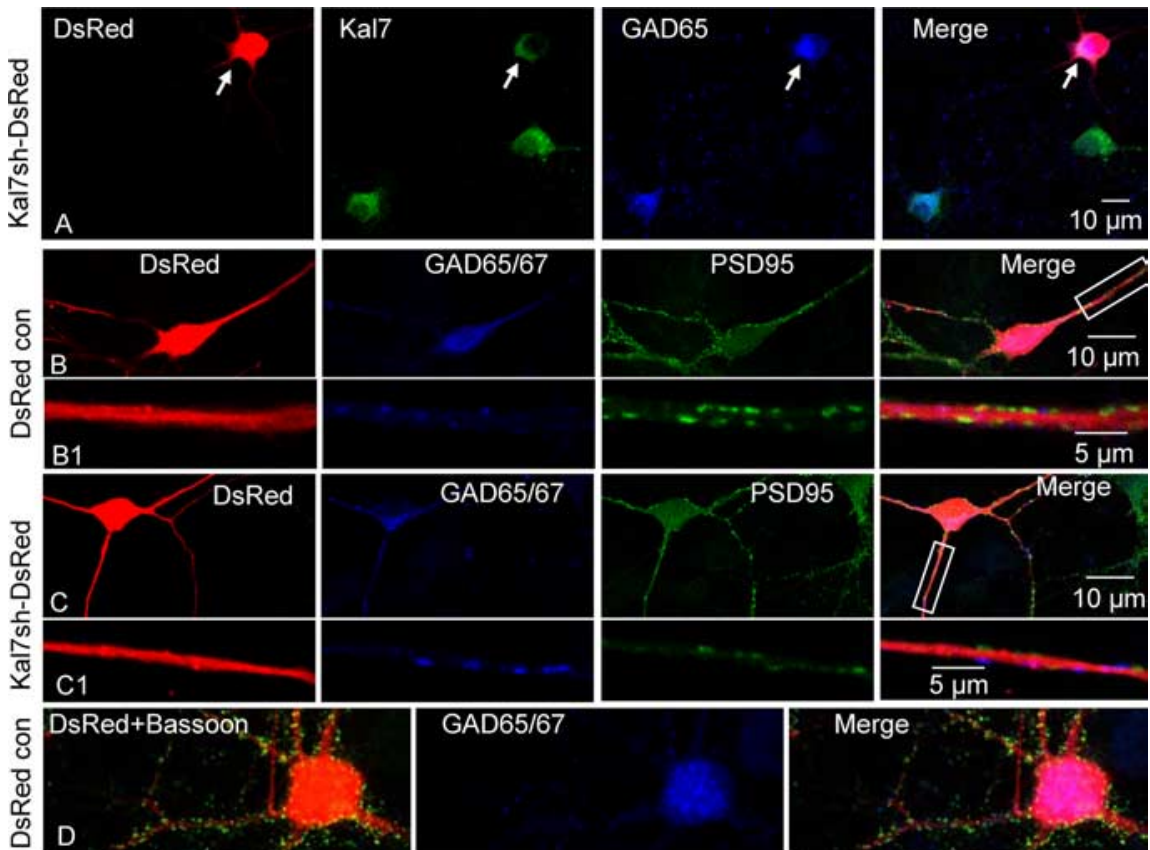

GAD65/67
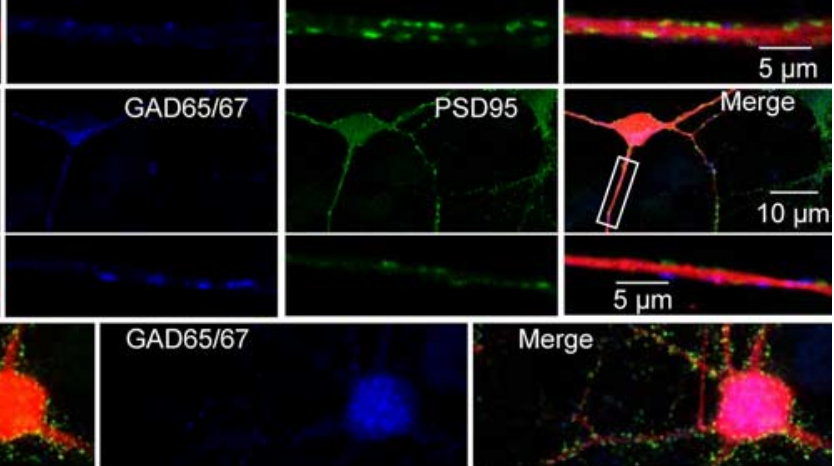

Figure 3. Expression of Kal7 shRNA causes a reduction in the number of excitatory synapses onto dendritic shafts of interneurons. Cultured hippocampal neurons (14 DIV) were transfected with pSIREN-Kal7 shRNA $(\boldsymbol{A}, \boldsymbol{C}, \boldsymbol{E}, \boldsymbol{F})$ or pSIREN-DsRed $(\boldsymbol{B}, \boldsymbol{D}) . \boldsymbol{A}$, Cells were stained at 20 DIV with antibodies specific for Kal7 (green) and GAD65 (blue); DsRed marks transfected neurons. Expression of Kal7 shRNA (arrow) caused a decrease in Kal7 staining $(\boldsymbol{A}, \mathbf{G} ; n=16)$ and did not alter Kal12 staining $(\mathbf{G} ; n=14)$. $B, C$, Cells were double stained with antibodies specific to GAD65/67 (blue) and PSD-95 (green). $B 1$ and $C 1$ are high-power images of $\boldsymbol{B}$ and $\boldsymbol{C}$, respectively. Expression of Kal7-shRNA reduced the number of PSD-95-positive clusters in dendrites $(\boldsymbol{C} \boldsymbol{H} ; \boldsymbol{H} \boldsymbol{n}=\mathbf{1 5})$. $D-F$, Cells were double stained with antibodies specific to bassoon (green) and GAD65/67 (blue); DsRed is red. Expression of Kal7-shRNA reduced the number of bassoon-positive presynaptic clusters $(\boldsymbol{H} ; n=15)$. However, the number of GAD65/67positive clusters along the dendrites was not altered $(\boldsymbol{H} ; n=12)$. Student's $t$ test, ${ }^{*} p<0.05$.

particles using the Helios Gene Gun System (Bio-Rad, Hercules, CA). After transfection $(48,72$, and $96 \mathrm{~h})$, slices were fixed and processed for immunostaining as described above. High-density cultures of dissociated hippocampal neurons were biolistically transfected with pCMSEGFP, pCMS-Kal7-antisense, pDsRed-Monomer-N1, pSIREN-Kal7 shRNA, or pSIREN-PAM shRNA at 14 DIV as described previously (Ma et al., 2003). Cells were fixed and processed for immunostaining $6 \mathrm{~d}$ after transfection.

\section{Image analysis and quantification}

Images captured using a Zeiss LSM510 confocal microscope were analyzed as described previously (Ma et al., 2003). For organotypic slices, only GFP-positive neurons in the CA1 area were analyzed; interneurons and pyramidal neurons were identified based on their locations and mor- 


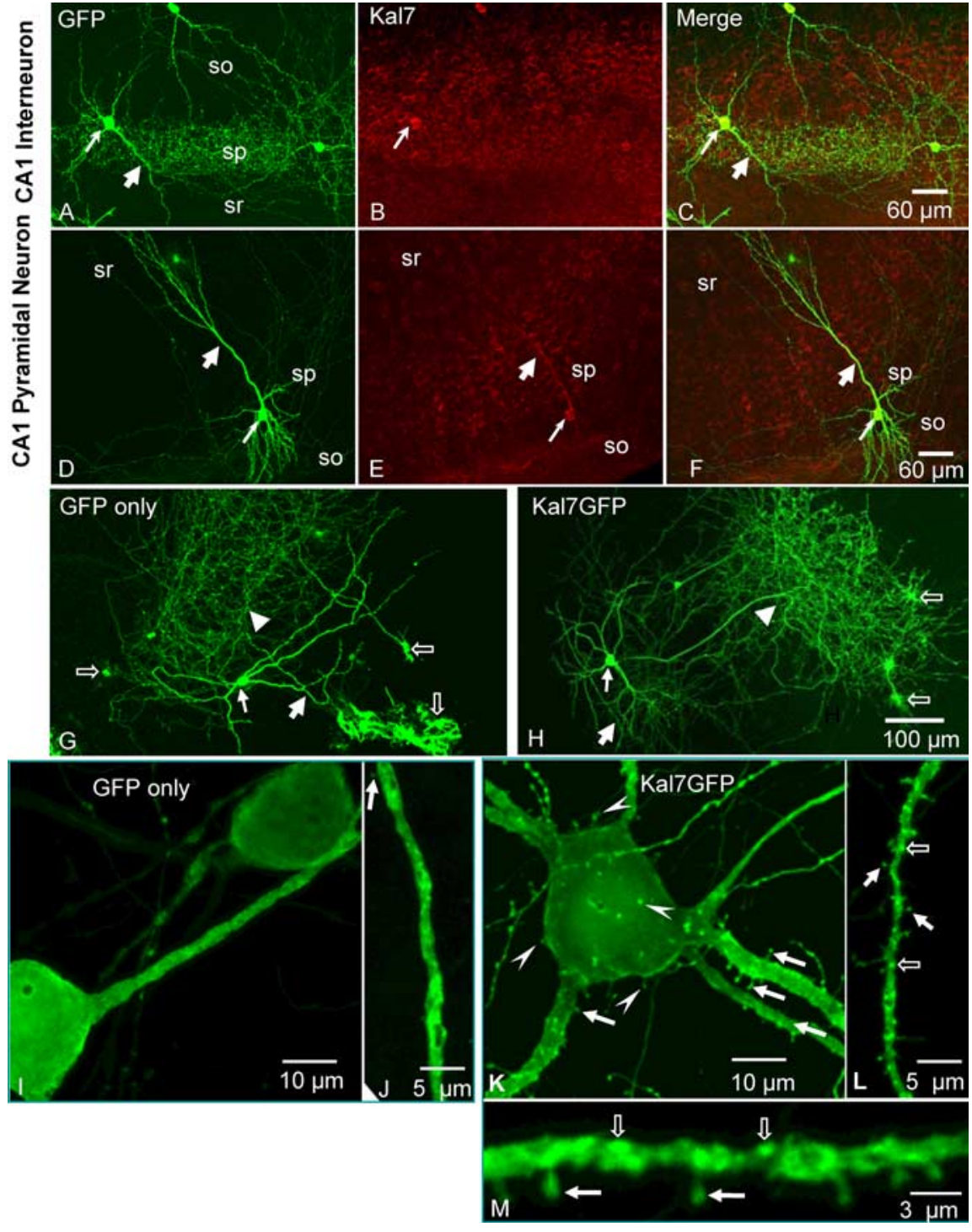

Figure 4. Expression of Kal7GFP alters the number of dendritic branches and spines in CA1 interneurons. Kal7GFP was expressed in $C A 1$ hippocampal interneurons $(\boldsymbol{A}-\boldsymbol{C})$ and pyramidal neurons $(\boldsymbol{D}-\boldsymbol{F})$. Hippocampal slices were biolistically transfected

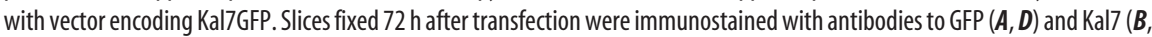
$\boldsymbol{E})$. Interneurons ( $\boldsymbol{A}-\boldsymbol{C}$, thin arrows) were distinguished from CA1 pyramidal neurons ( $\boldsymbol{D}-\boldsymbol{F}$, thin arrows) based on morphology and localization. Thick arrows, Dendrites; so, stratum oriens; sr, stratum radiatum; sp, stratum pyramidal. $\boldsymbol{G}, \boldsymbol{H}$, Interneurons (thin arrows) and glia (open arrows) from the CA1 area of hippocampal slices were biolistically transfected with vectors encoding GFP $(\boldsymbol{G})$ or Kal7GFP $(\boldsymbol{H})$. Thick arrows, Dendrites; arrowheads, axons. $\boldsymbol{I}-\boldsymbol{K}$, High-power images, showing GFP-positive interneuron is free of spines, and Kal7GFP-positive interneuron contains spines in soma (arrowheads) and dendrite (solid arrows). Some spines are mushroom structures with clear heads ( $L, M$, solid arrows); Kal7GFP-positive clusters are also found within the dendritic shaft ( $L, M$, open arrows).

phology. For dissociated hippocampal neurons, GFP-positive interneurons were identified by GAD staining (Esclapez et al., 1994). Z-stacks (Z step, $1 \mu \mathrm{m}$ ) were taken using a $20 \times$ objective ( 0.7 digital zoom factor); images of the entire neuron were generated with Zmaris 3.2 software (Bitplane, Zürich). Dendrites were traced, total dendritic length and dendritic branch segments were calculated, and Sholl profiles were analyzed using Neurolucida (Ma et al., 2003).

For quantification of spine density and synaptic clusters, a stack of images ( $Z$ step, $0.3 \mu \mathrm{m}$ ) was acquired using a $63 \times$ objective $(2.5$ digital zoom factor), and dendrites were visualized in three-dimension (Ma et al., 2003). For analysis of colocalization of Kal7GFP clusters and synaptic markers, single plane images through the brightest point were used. For each experiment, all images were taken with identical settings under the same conditions. Spine density and synaptic clusters were counted after images were calibrated, and thresholds were set to ensure that all inter- esting structures were included in the analysis. Quantifications were performed using MetaMorph (Molecular Devices, Downingtown, PA) and were limited to dendrites within 100 $\mu \mathrm{m}$ of the soma. The length of each traced spine was measured using MetaMorph. Data are presented as average \pm SEM. Statistical analyses were performed with JMP6 software (SAS Institute, Cary, NC) using one-way ANOVA followed by Dunnett's test to assess statistical significance between groups $\left({ }^{*} p<\right.$ 0.05 or ${ }^{\star *} p<0.01$ was considered statistically significant).

\section{Results}

Kal7 is expressed in hippocampal interneurons and localized at excitatory synapses

Antisera specific for the unique $\mathrm{C}$ terminus of Kal7 also detect $\Delta \mathrm{Kal} 7$, a splice variant generated from a different promoter (Fig. 1A). Because Kal7 protein is much more prevalent than $\Delta \mathrm{Kal} 7$ (McPherson et al., 2002), we simply refer to cross-reactive material as Kal7. Interneurons are very heterogeneous, and we focus here on interneurons in the CA1 region that can be identified in coronal sections of adult rat hippocampus based on their location and staining with antibody to GAD65/67 (Fig. $1 B-D 1)$, a marker for inhibitory interneurons, or PV (Fig. 1E-G1) (Freund and Buzsaki, 1996). Two different antibodies to GAD were used to identify GABAergic neurons. The polyclonal antibody to GAD65/67 labels both presynaptic GABAergic endings and the soma of GABAergic neurons (Esclapez et al., 1994). Because the monoclonal antibody is specific for GAD65, it visualized presynaptic endings more readily than the cell soma (Chang and Gottlieb, 1988).

Kal7-positive interneurons are scattered throughout the stratum oriens, stratum pyramidal, and stratum radiatum (Fig. $1 B-D$, arrows). Of the 128 GAD65/ 67-positive interneurons examined, all contained Kal7; levels were generally lower than in CA1 pyramidal neurons. Of the 98 parvalbumin-positive interneurons examined, all contained Kal7; levels were lower than in the soma of most parvalbumin negative neurons. (Fig. 1E-G1). The level of Kal7 in the dendrites extending from the soma of parvalbuminpositive interneurons is much lower than in the soma (Fig. 1E1-G1).

To evaluate Kal7 staining in the dendrites of mature interneurons, 21 DIV cultures of dissociated hippocampal neurons were fixed with methanol and stained for Kal7 and GAD65/67. Kal7 staining was observed in the soma and in the dendrites of both interneurons and other neurons (Fig. $2 A-F$ ). However, Kal7 levels in the dendrites of interneurons are lower than in other neurons (Fig. $2 A, D$ ). Kal7 staining in the dendrites of GAD65/67-positive interneurons appears in clusters (Fig. 
2D1); the Kal7-positive clusters are not colocalized with GAD65/67, which is present in presynaptic inhibitory terminals (Fig. 2D1-F1).

To determine whether the Kal7-positive clusters along the dendrites of interneurons identified by GAD65/67 staining were synaptic structures, Kal7 and a variety of synaptic markers were visualized simultaneously: Bassoon, a presynaptic terminal marker; VGLUT1, a marker for glutamatergic presynaptic endings; PSD-95, a post-synaptic density marker; NMDA receptor subunit NR1 and AMPA receptor subunit GluR2, markers for the postsynaptic side of excitatory synapses. Kal7-positive clusters were apposed to almost all VGLUT1-positive clusters (Fig. 2G-I) and to most Bassoon-positive clusters (Fig. $2 J-L$ ) along interneuron dendrites. Bright Kal7-positive clusters were always-positive for PSD-95 (Fig. $2 M-O$ ) and NR1 (Fig. $2 P-R$ ). Although a subset of the Kal7-positive clusters $(13 \pm 1 \%)$ were positive for GluR2, most ( $95 \pm 4 \%$ ) of the GluR2-positive clusters were positive for $\mathrm{Kal7}$ (Fig. 2S-U). These data indicate that Kal7 in interneurons is localized to the postsynaptic side of excitatory synapses onto the dendritic shaft.

\section{Reduced endogenous Kal7 expression causes a decrease in excitatory synapses onto interneurons}

To determine whether Kal7 plays an essential role in the formation of excitatory synapses onto the dendritic shafts, we sought a means of selectively reducing expression of this isoform of Kalirin. Antisense-mediated reductions in the expression of all of the major isoforms of Kalirin in hippocampal pyramidal neurons revealed an essential role for Kalirin in maintenance of the dendritic arbor and dendritic spines (Ma et al., 2003). Kal7 is the major isoform in the adult brain (Johnson et al., 2000), and its appearance at the time of synapse formation suggests a specific role for this isoform in synaptogenesis. Kal7 immunoreactivity in CA1 interneurons and pyramidal cells was first detected at P7, with increasing levels of staining in both cell types from P7 to P30 (data not shown). A Kal7/ $\Delta$ Kal7-specific shRNA targeted to the unique 3 - -untranslated region of $\mathrm{Kal} / \Delta \mathrm{Kal} 7$ was expressed in dissociated hippocampal neurons (Fig. 3A). Expression of Kal7 shRNA caused a decrease in endogenous Kal7 immunoreactivity in GAD65-positive interneurons compared with neighboring nontransfected interneurons (Fig. 3A, G); staining for Kal12 was not affected, demonstrating specificity. Expression of Kal7 antisense RNA (pCMS-Kal7-antisense) produced effects identical to those of Kal7 shRNA (data not shown). To address specificity, we created an shRNA vector targeted to PAM, a transcript highly expressed in hippocampal interneurons (Ma et al., 2002). Expression of PAM shRNA decreased PAM immunoreactivity by $56 \%$ in GAD65-positive interneurons but did not alter the number of PSD-95 clusters along their dendrites (data not shown).

Dissociated hippocampal neurons transfected with control vector (pSiren.DsRed) or pSiren-Kal7 shRNA were fixed after $6 \mathrm{~d}$ and simultaneously visualized with antibodies to GAD65/67 and either PSD-95 (Fig. 3 B, C) or bassoon (Fig. 3D-F). Expression of Kal7 shRNA caused a decrease in the density of PSD-95 clusters along the dendrites of interneurons (Fig. 3B1,C1,H). Similarly, the density of presynaptic bassoon-positive clusters along the dendrites of interneurons expressing the Kal7-shRNA is decreased when compared with control DsRed expressing interneurons (Fig. $3 D, E, H$ ) or neighboring nontransfected interneurons (Fig. $3 F$ ). The prevalence of GAD65/67-positive clusters along the dendrites of Kal7-shRNA expressing interneurons was not altered (Fig. $3 H$ ). Kal7 thus plays an essential role in the formation of excitatory synapses on spines and dendritic shafts.
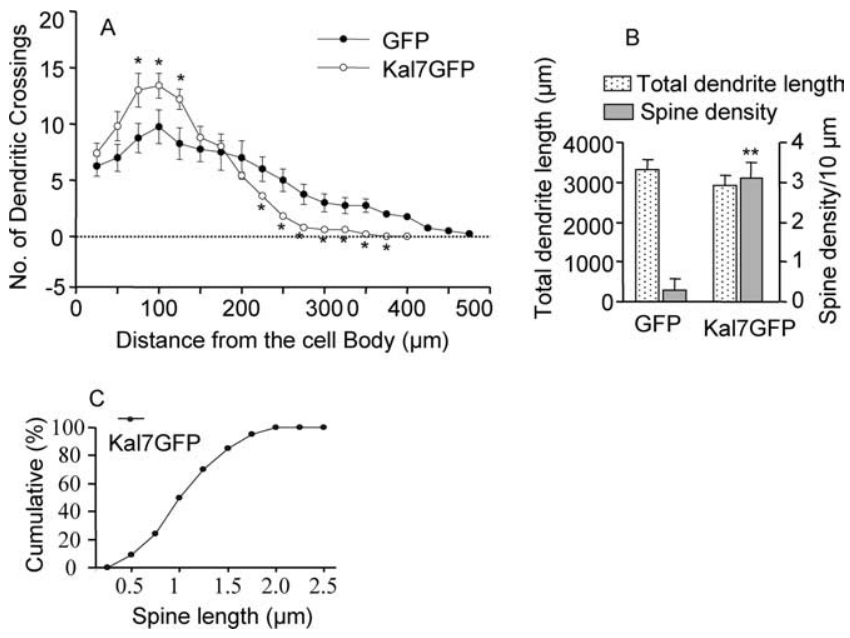

Figure 5. Expression of Kal7GFP alters the number of dendritic branches and spines in CA1 interneurons. $\boldsymbol{A}$, Sholl analysis ( $n=13$ for GFP; $n=12$ for Kal7GFP). $\boldsymbol{B}$, Left $y$-axis, Total dendritic length in CA1 interneurons. $\boldsymbol{B}$, Right axis, Spine density in CA1 interneurons (GFP, $0.28 \pm 0.31 / 10 \mu \mathrm{m}, n=13 ; \mathrm{Kal} 7 \mathrm{GFP}, 3.10 \pm 0.40 / 10 \mu \mathrm{m}, n=12)$. C, Cumulative frequency plot of spine length in neurons expressing Kal7GFP (800 spines in 12 interneurons). One-way ANOVA followed by Dunnett's test to assess statistical significance between groups $(\boldsymbol{A})$. Student $t$ test $(\boldsymbol{B}),{ }^{*} p<0.05,{ }^{* *} p<0.01$.

\section{Overexpression of Kal7 increases dendritic branching and spine formation in hippocampal interneurons}

Because endogenous Kal7 is essential for excitatory synapses on the dendritic shafts, we wondered whether overexpression of Kal7 would cause the formation of spine-like structures or increase the number of shaft synapses. To determine the effects of slightly increasing Kal7 levels in interneurons, hippocampal slices were biolistically transfected with vectors encoding GFP or bioactive Kal7GFP (Fig. S2, available at www.jneurosci.org as supplemental material) (Fig. 4). The use of organotypic slices facilitates identification of CA1 interneurons and pyramidal neurons. Slices prepared from P9 pups were transfected at 2 DIV and were analyzed 48, 72, and 96 h later. GFP was visualized using an antibody to intensify the signal and ensure visualization of all processes. The validity of this approach was established by quantifying dendritic spines in interneurons simultaneously expressing GFP and MycKal7; identical results were obtained using GFP staining (turning the red channel off and visualizing only the green channel) or Myc staining (turning the green channel off and visualizing only the red channel) to identify dendritic spines (Fig. S3, available at www.jneurosci.org as supplemental material). Similarly, the large increase in spine density produced by MycKal7 (visualized with cotransfected GFP) was identical to the increase seen using Kal7GFP (Fig. S3, available at www. jneurosci.org as supplemental material). Based on neuronal morphology and the intensity of the Kal7GFP signal, $72 \mathrm{~h}$ posttransfection was selected as the optimal time for analysis; all slices in this experiment were analyzed at this time (Fig. 4). Interneurons can be distinguished from pyramidal neurons according to their localization and their short, thick dendrites with long, thin, complex axons (Fig. 4D,G,H). Compared with nontransfected, neighboring neurons $(n=22)$, expression of Kal7GFP $(n=20)$ results in a twofold increase in Kal7 immunoreactivity in processes and a 2.5-fold increase in the cell soma (Fig. 4). Kal7GFP is observed in interneurons and pyramidal neurons (Fig. $4 A-H$ ) as well as in glia (Fig. 4G,H, open arrows) after biolistic transfection.

Interneurons in the CA1 region expressing GFP (Fig. 4G) or Kal7GFP (Fig. $4 H$ ) were subjected to Sholl analysis. Expression of 

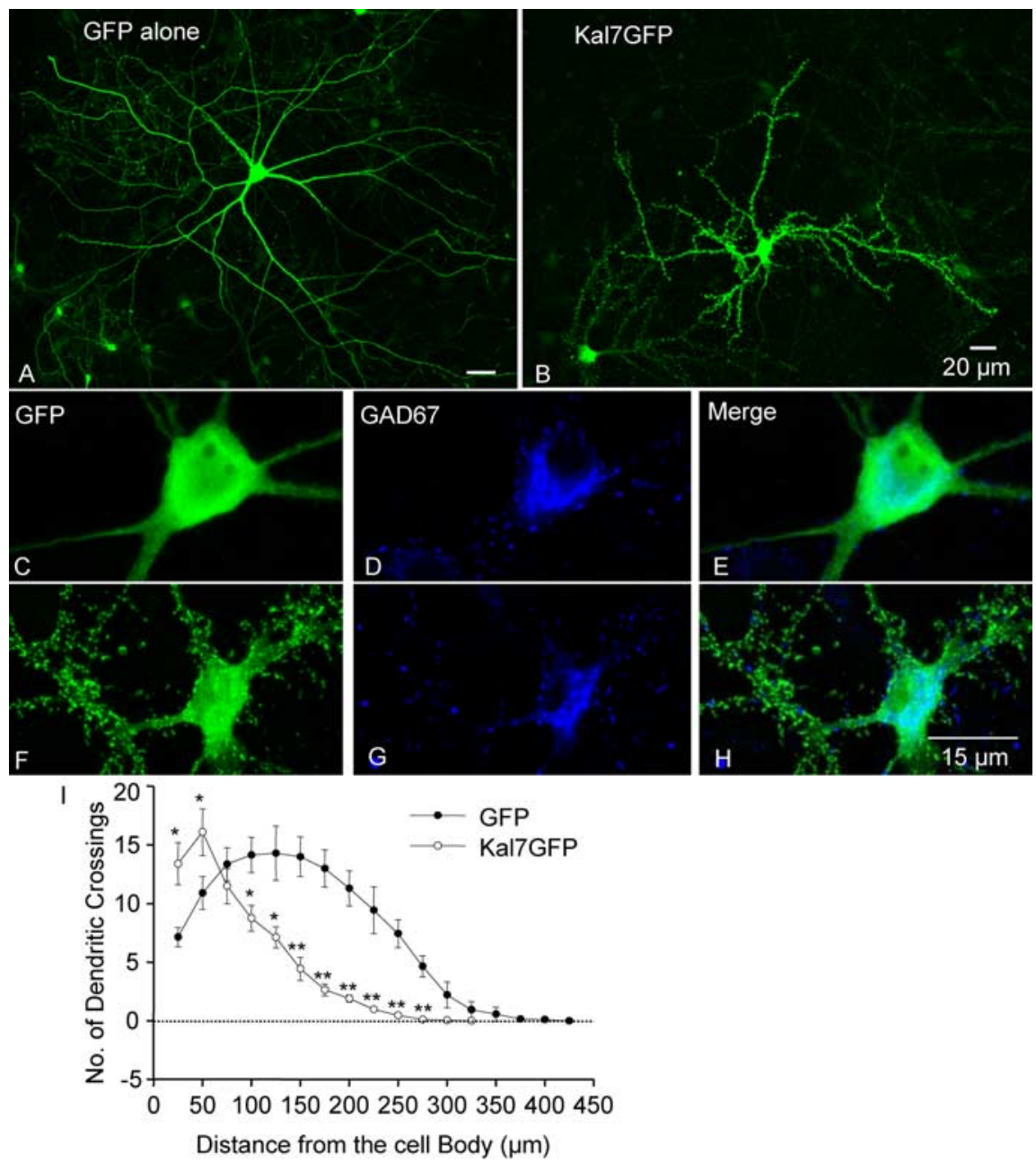

Figure 6. Expression of Kal7GFP alters dendritic morphology in cultured hippocampal interneurons. Hippocampal neurons in dissociated culture were transfected with vectors encoding $\operatorname{GFP}(\boldsymbol{A}, \boldsymbol{C}-\boldsymbol{E})$ or Kal7GFP $(\boldsymbol{B}, \boldsymbol{F}-\boldsymbol{H})$ at 1 DIV. Neurons were triple stained at 16 DIV with antibodies to GFP $(\boldsymbol{A}, \boldsymbol{B}), \operatorname{MAP2}$ (data not shown), and $\operatorname{GAD} 65 / 67(\boldsymbol{D}, \boldsymbol{E}, \boldsymbol{G}, \boldsymbol{H}) . \boldsymbol{C}-\boldsymbol{E}$ and $\boldsymbol{F}$ - $\boldsymbol{H}$ are high-power images from $A$ and $B$, respectively. Sholl analysis $(I)(n=17$ for GFP; $n=14$ for Kal7GFP). One-way ANOVA followed by Dunnett's test to assess statistical significance between groups $(\boldsymbol{I}),{ }^{*} p<0.05,{ }^{* *} p<0.01$.

Kal7GFP in interneurons increased the number of dendritic branches within $125 \mu \mathrm{m}$ of the cell soma (Fig. $5 \mathrm{~A}$ ); total dendritic length was unaltered (Fig. $5 B$ ). The total number of dendrites was unaltered after expression of Kal7. Exogenous Kal7GFP is found in long, highly branched axons, which were not analyzed because of their complexity (Fig. $4 H$ ).

To evaluate the possibility that expression of Kal7GFP might induce formation of spine-like structures in hippocampal interneurons, biolistically transfected neurons were systematically analyzed at higher magnification (Fig. $4 I-M$ ). As expected, the dendrites of GFP-expressing interneurons (controls) were not decorated with spine-like structures, and spine-like structures were never observed on their soma (Fig. 4I,J). Overexpression of Kal7GFP in interneurons results in the appearance of spine-like structures along their dendrites and around their soma (Fig. $4 \mathrm{~K}-$ $M)$. Some of these spine-like structures have the appearance of mushroom spines, suggesting they are mature, functional spines (Fig. $4 L, M$ ). In addition, Kal7GFP-positive clusters are observed along the dendritic shaft (Fig. $4 L, M$, open arrows). Quantification revealed a $>10$-fold increase in dendritic spine density after expression of Kal7GFP in hippocampal interneurons (GFP, $0.28 \pm 0.31 / 10 \mu \mathrm{m} ; \mathrm{Kal}$ GFP, $3.10 \pm 0.40 / 10 \mu \mathrm{m})$. The length of these spines is not longer than $2 \mu \mathrm{m}$, and most of them are between 0.5 and $1.75 \mu \mathrm{m}$ (Fig. 5C).

\section{Overexpression of Kal7GFP alters dendritic morphology and induces spine formation in hippocampal interneurons in culture}

To determine whether overexpression of Kal7 can alter dendritic morphology and induce spine formation in interneurons in culture, dissociated cells were transfected with vectors encoding GFP or Kal7GFP. To identify interneurons, cultures were stained simultaneously with antibodies to GAD65/67 (Esclapez et al., 1994) (Fig. 6C$H)$, MAP2 to distinguish dendrites from axons (data not shown) and GFP (Fig. 6A$H)$. As observed in slice cultures, expression of Kal7GFP increases the number of dendritic branches within $50 \mu \mathrm{m}$ of the cell soma and decreases the number of long dendrites (>100 $\mu \mathrm{m}$ ) (Fig. 6I). Unlike the response observed in organotypic slices, total dendritic length is diminished in Kal7GFP expressing interneurons in dissociated culture (GFP, $3504 \pm 165 \mu \mathrm{m}$; GFPKal7, $2112 \pm 121 \mu \mathrm{m} ; p<0.01$, Student's $t$ test). To identify NPY-expressing interneurons, neurons were triple stained simultaneously with antibody to GFP, NPY, and GAD65 (Fig. 7A-H). NPY is expressed in hippocampal interneurons under basal conditions (Ma et al., 2002). To identify parvalbumin-expressing interneurons (Gulyas et al., 1999), neurons were double stained with antibodies to parvalbumin and GFP (Fig. 7I,J). To quantify spine density, neurons stained with antibodies to GFP and GAD65/67 were stained simultaneously for MAP2 to highlight dendritic shafts (Fig. $7 L, M$ ) (Fig. S4, available at www.jneurosci.org as supplemental material). Spine formation in dissociated cultures was examined using higher-power images. As observed in organotypic slices, the dendrites of interneurons expressing GFP are almost free of spine-like structures (Fig. $7 A, L$ ) (Fig. S4A, available at www.jneurosci.org as supplemental material). Spine-like structures are never found on the somas of interneurons expressing GFP (Fig. 7A) (Fig S4A, available at www.jneurosci.org as supplemental material). Expression of Kal7GFP induces a marked increase in spine-like structures along the dendrites and on the somas of interneurons (Fig. 7 E, J,J1,M) (Fig. S4 H, I, available at www.jneurosci.org as supplemental material). Most of these spines are $0.5-2.5 \mu \mathrm{m}$ long (Fig. $7 N$ ). In GFP-expressing interneurons, GFP is diffusely merged with staining for MAP2 (Fig. 7L) (Fig. S4D, available at www. jneurosci.org as supplemental material). In contrast, in Kal7GFP-expressing interneurons, the GFP occurs in clusters both within the dendritic shaft and at the tips of dendritic spines (Fig. 7M) (Fig. S4H, available at www.jneurosci.org as supplemental material). Based on immunostaining, Kal7GFP is again being expressed in dendrites at levels that are 1.9-fold higher than endogenous levels of Kal7. Quantification reveals a far higher 
number of dendritic spine-like structures formed in response to Kal7GFP in cell culture $(15.0 \pm 0.7 / 10 \mu \mathrm{m})$ than in slice culture $(3.1 \pm 0.4 / 10 \mu \mathrm{m})$. The Kal7-induced spines formed in culture tend to be longer than the spines formed in slice cultures (Fig. $7 N$ ).

To ensure that Kal7GFP and MycKal7 have similar effects on spine formation, dissociated hippocampal neurons were transfected with vector encoding soluble GFP alone, cotransfected with vectors encoding soluble GFP and MycKal7, or transfected with vector encoding Kal7GFP alone. Coexpression of MycKal7 with GFP or expression of Kal7GFP alone caused a similar increase in spine-like structures in hippocampal interneurons (Fig. S3, available at www.jneurosci.org as supplemental material).

The Kal7GFP-positive spines are sites of excitatory synapses in interneurons

Kal7GFP clusters are localized to spine tips and within the dendritic shaft of interneurons. To determine the characteristics of these Kal7GFP clusters, hippocampal neurons expressing GFP (Fig. 8A,a) or Kal7GFP (Fig. $8 A, b$ ) were visualized simultaneously with antibodies to GFP, PSD-95, and GAD65/67. In interneurons expressing GFP, all of the PSD-95-positive clusters are localized within the dendritic shaft (Fig. 8A,a1); no PSD-95-positive spine-like structures are present. In contrast, in Kal7GFP-expressing interneurons, PSD-95-positive clusters are observed both within the dendritic shaft and at the tips of spine-like structures (Fig. $8 A, b 1)$. Colocalization analysis indicates that $97 \%$ of the Kal7GFP clusters are also stained for PSD-95. The number of PSD95-positive clusters within the dendrites or in spine-like structures was quantified. Compared with control interneurons expressing GFP, expression of Kal7GFP causes a twofold increase in the linear density of dendritic PSD-95-positive clusters (Fig. 8C). Classifying total PSD-95positive clusters as within the dendritic shaft or in spine-like structures revealed that $96 \%$ were in the dendritic shaft in GFP expressing interneurons, whereas only $55 \%$ were in the dendritic shaft in interneurons expressing Kal7GFP.

PSD-95 is localized to glutamatergic synapses on spines and shafts and is involved in the formation of excitatory synapses (Hunt et al., 1996; El Husseini et al., 2000). To evaluate the presence of presynaptic endings, cultures expressing GFP (Fig. $8 B, c$ ) or Kal7GFP (Fig. $8 B, d$ ) were stained simultaneously with antibodies to GFP, bassoon, and GAD65/67. In interneurons expressing GFP, bassoon-positive presynaptic terminals are directly apposed to the dendritic shaft (Fig. 8c1). In Kal7GFP-expressing interneurons, almost every Kal7GFP cluster is aligned with a bassoon-positive presynaptic terminal (Fig. 8d1). Presynaptic
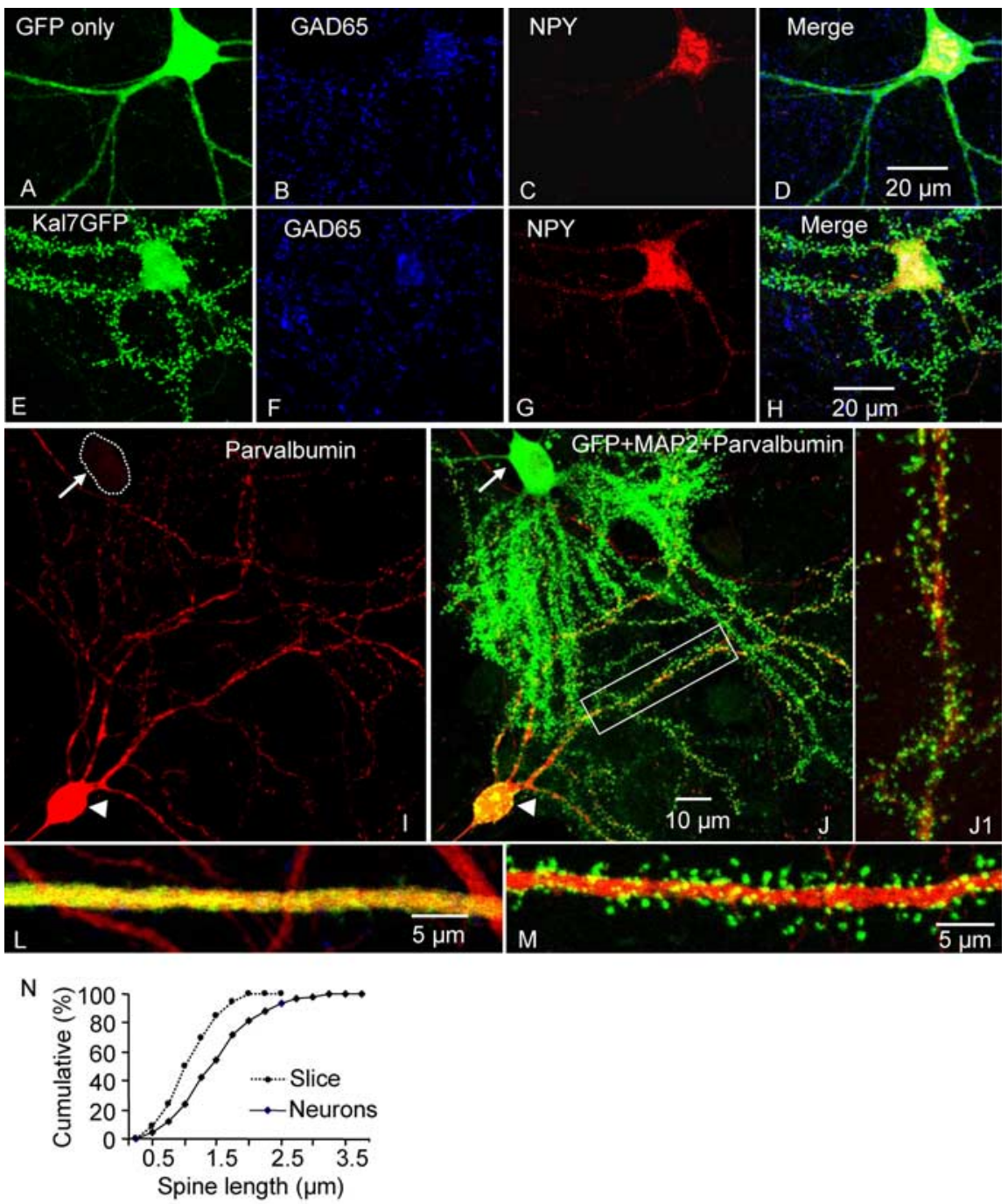

Figure 7. Overexpression of Kal7 forces aspiny interneurons to produce spine-like structures. Hippocampal neurons in dissociated culture were transfected with vector encoding GFP alone $(\boldsymbol{A}, \boldsymbol{L})$ or $\operatorname{Kal} 7 \mathrm{GFP}(\boldsymbol{E}, \boldsymbol{J}, \boldsymbol{M})$ at 1 DIV. $\boldsymbol{A}-\boldsymbol{H}$, Cultures were triple stained with antibodies to $\operatorname{GFP}(\boldsymbol{A}, \boldsymbol{E}$, green), GAD65 ( $\boldsymbol{B}, \boldsymbol{F}$, red), and NPY $(\boldsymbol{C}, \boldsymbol{G}$, blue). Merged images are shown in $\boldsymbol{D}$ and $\boldsymbol{H}$. $\boldsymbol{I}, \boldsymbol{J}$, Cultures were double stained with antibodies to parvalbumin $(\boldsymbol{I}$, red) and GFP (green in merged image $\boldsymbol{J})$. $\boldsymbol{J} \mathbf{1}$ is high-power image ( 年 neurons transfected with Kal7GFP (1000 spines in 15 interneurons were examined). For comparison, the dashed line repeats cumulative frequency plot of spine length in slices transfected with Kal7GFP (Fig. 4R). Arrows show parvalbumin-negative (I, dashed circle) cell soma, whereas arrowheads show parvalbumin-positive cell soma.

endings terminate on Kal7GFP-positive clusters that occur within the dendritic shaft and at the tips of spine-like structures (Fig. 8d1, arrowheads and arrows, respectively). Expression of Kal7GFP causes a twofold increase in the total number of bassoon-positive presynaptic terminals along the dendrites of interneurons (Fig. 8C), matching the increase of PSD-95 clusters.

In addition to excitatory input, the dendrites of interneurons receive inhibitory inputs from other GABAergic interneurons (Cobb et al., 1997). Inhibitory synapses were identified using antibody to GAD65/67. The dendrites of control interneurons were lined with GAD65/67-positive structures, which correspond to inhibitory synapses (Fig. 8a1,c1). As expected, GAD65/ 67-positive clusters overlap with a subset of the Bassoon-positive clusters; GAD65/67-positive inhibitory synapses are much less 

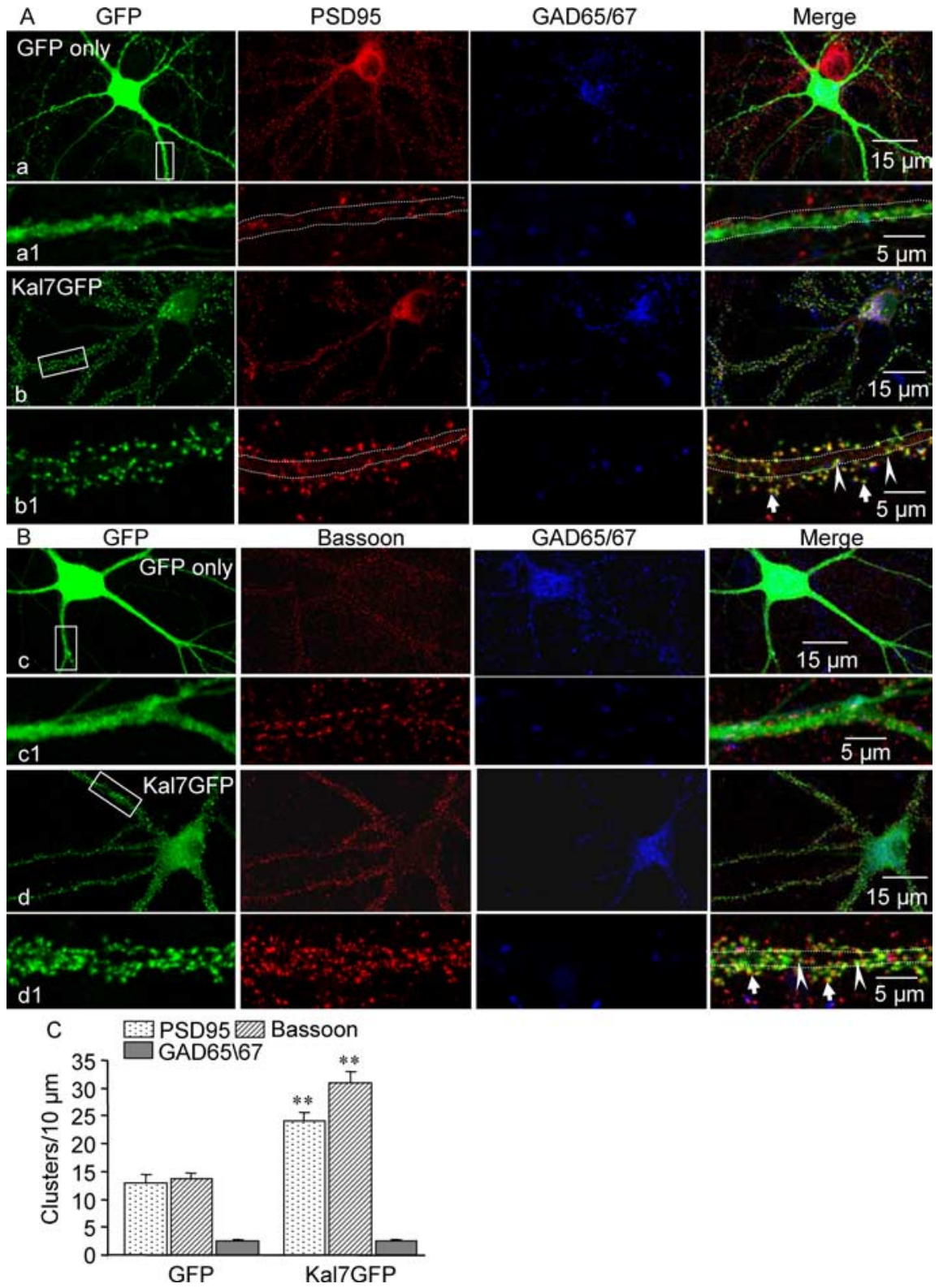

Figure 8. Kal7GFP is localized to synapses in interneurons. $\boldsymbol{A}$, Cultured hippocampal neurons were transfected with vectors encoding GFP $(\boldsymbol{a})$ or Kal7GFP $(\boldsymbol{b})$. As indicated, at 16 DIV, neurons were triple stained with antibodies to GFP, PSD-95, and GAD65/67. The strongly PSD-95-positive neuron in a was not stained for GAD65/67 and is not an interneuron. High-power images of boxed areas are shown below $(\boldsymbol{a} \mathbf{1}, \boldsymbol{b} \mathbf{1}) . \boldsymbol{B}$, Cultured hippocampal neurons were transfected with vectors encoding GFP alone (c) or Kal7GFP (d), and cells were triple stained with antibodies to GFP, bassoon, and GAD65/67. High-power images of boxed areas are shown below $(\boldsymbol{c} \mathbf{1}, \mathbf{d} \mathbf{1})$. C, Density of dendritic PSD-95 clusters $(n=16)$, bassoon-positive $(n=16)$, and GAD65/67-positive $(n=14)$ presynaptic endings on the dendrites of these interneurons were quantified. Student's $t$ test, ${ }^{* *} p<0.01$. Arrowheads show synaptic clusters in the dendritic shaft, whereas arrows show synaptic clusters at the tips of spine-like structures.

prevalent than PSD-95-positive clusters (Fig. 8a1). Expression of Kal7GFP did not alter the number of GAD65/67-positive presynaptic endings along the dendrites of interneurons (Fig. 8b1, d1,C). Kal7GFP-positive clusters rarely aligned with GAD65/67positive presynaptic terminals (Fig. $8 b 1, d 1$ ).

\section{Localization of Kal7GFP at excitatory synapses of mature interneurons}

To further characterize the Kal7GFP clusters formed in interneurons (identified using antisera to GAD65 or GAD65/67), cultures transfected at 1 DIV and maintained in culture for 3 weeks were visualized with antibodies to GFP and to the vesicular GABA transporter, a marker for inhibitory GABAergic presynaptic endings, or VGLUT1 (Fig. 9). VGLUT1-positive excitatory presynaptic terminals are localized to the surface of the dendritic shaft in control interneurons expressing GFP (Fig. 9A). VGLUT1-positive excitatory presynaptic terminals are localized to the tips of the spine-like structures formed in response to expression of Kal7GFP and along the dendritic shaft (Fig. 9B). Quantitative analysis reveals that $96 \%$ of the Kal7GFP-positive clusters are aligned with VGLUT1-positive clusters along the dendrites of interneurons expressing Kal7GFP (Fig. 9I). In contrast, little colocalization of Kal7GFP-positive clusters and VGATpositive (Fig. 9C,I) or GAD65-positive (Fig. 9D,I) inhibitory presynaptic terminals was observed.

We next asked whether the clusters of Kal7GFP in the dendrites of hippocampal interneurons colocalized with excitatory glutamate receptors (NR1, GluR1, or GluR2) or with inhibitory $\mathrm{GABA}_{\mathrm{A}}$ receptors (Fig. 9E-H). Almost all (96\%) of the Kal7GFP-positive clusters colocalized with NR1-positive clusters (Fig. 9E,J). A slightly smaller percentage $(89 \%)$ colocalized with GluR1-positive clusters (Fig. 9F,J). Most (96\%) colocalized with GluR2-positive clusters (Fig. 9G,J). In contrast, only $1 \%$ of the Kal7GFP-positive clusters overlap with $\mathrm{GABA}_{\mathrm{A}}$ receptor-positive clusters (Fig. $9 H, J)$. PSD-95, which is present at excitatory endings on spines and on dendritic shafts, is present at $98 \%$ of the Kal7GFPpositive clusters (Fig. 9J).

PDZ binding motif is essential for Kal7induced spine formation, and overexpression of PSD-95 does not make aspiny interneurons produce spine-like structures

The C-terminal of Kal7 interacts with several PDZ domain proteins localized to spine-like structures (Penzes et al., 2001b). To determine whether the PDZ binding motif of Kal7 is required for its effect on spine formation in interneurons, neurons were cotransfected with DsRed plus Kal7 $\Delta \mathrm{CT}$, which lacks the PDZ binding motif, at $\mathrm{P} 1$ and examined at P16. Expression of Kal7 $\Delta \mathrm{CT}$ did not cause spine formation in interneurons; myc staining was diffuse in the cell soma and dendritic shafts (Fig. $10 A-D$ ). Of the 20 myc-positive interneurons examined, all showed smooth dendritic shafts. As expected, expression of Kal7 in parallel cultures caused spine formation in interneurons (data not shown).

Because the PDZ binding motif of Kal7 is essential, we next asked whether overexpression of one of its targets, PSD-95, might reproduce the effect of Kal7. Hippocampal neurons in dissociated culture were transfected with vector encoding 
PSD-95 at 1 DIV, and neurons were doubly stained at 18 DIV with antibodies to PSD-95 and GAD65/67 (Fig. 10). The levels of PSD-95 in PSD-95-transfected interneurons are $\sim 11$ times higher than in nontransfected interneurons. Exogenous PSD-95 did not cause spine formation in hippocampal interneurons (Fig. $10 E, H)$. In the 18 PSD-95-positive interneurons examined, dendritic PSD-95 clusters were always localized on dendritic shafts. Interneurons expressing lower levels of exogenous PSD-95 also failed to develop spine-like structures (data not shown).

\section{Interruption of Kal7 PDZ binding motif} interactions blocks Kal7-induced formation of spine-like structures

Because the Kal7 PDZ binding motif is necessary for spine formation in interneurons, we sought a means of disrupting its interactions. Cell-permeant peptides offer a means of disrupting specific proteinprotein interactions in a controlled manner (Aarts et al., 2002). Wild-type and mutant cell-permeant Kal-7 C-terminal peptides were designed; R7-Kal7CT is detected by our polyclonal Kal7 antibody but not by our monoclonal Kal7 antibody (Fig. S5A-C, available at www.jneurosci.org as supplemental material). Neurons incubated with R7-Kal7CT (10 $\mu \mathrm{M})$ exhibited readily detectable staining in the soma, dendrites, and synaptic structures, indicating intracellular peptide uptake (Fig. S5A-E,H, available at www.jneurosci.org as supplemental material). Neurons treated with mutant R7-Kal7CT peptide showed staining in the cell soma (Fig. $\mathrm{S} 5 F$ ), with less peptide binding in dendrites (Fig. S5F, available at www. jneurosci.org as supplemental material). Cultures treated with vehicle revealed endogenous Kal7 staining in the soma (Fig. S5G, available at www.jneurosci.org as supplemental material).

To determine the effect of R7-Kal7CT on Kal7-induced spine formation, dissociated hippocampal neurons transfected with vector encoding Kal7GFP at 1 DIV were allowed to mature for 18 DIV. R7-Kal7CT, R7-Kal7CTmutant, or vehicle were added to the cultures at 7 and 13 DIV, and cultures were examined at 18 DIV. Staining with antibodies to GFP, MAP2, and GAD65 revealed the expected spine-like structures along the dendrites of GFP-Kal7-expressing neurons (Fig. $11 A-D, I)$. The R7-Kal7CT-treated neurons appeared to be healthy; MAP2-stained dendrites were smooth and uniformly stained. The R7-Kal7CT peptide blocked Kal7-induced spine formation compared with control neurons treated with the R7-Kal7CT mutant peptide (Fig. 11) (spine density, $13.2 \pm$ $1.2 / 10 \mu \mathrm{m}$ for mutant peptide, $1.8 \pm 0.9 / 10 \mu \mathrm{m}$ for Kal7CT peptide), which differs at only one residue from the R7Kal7CT peptide.
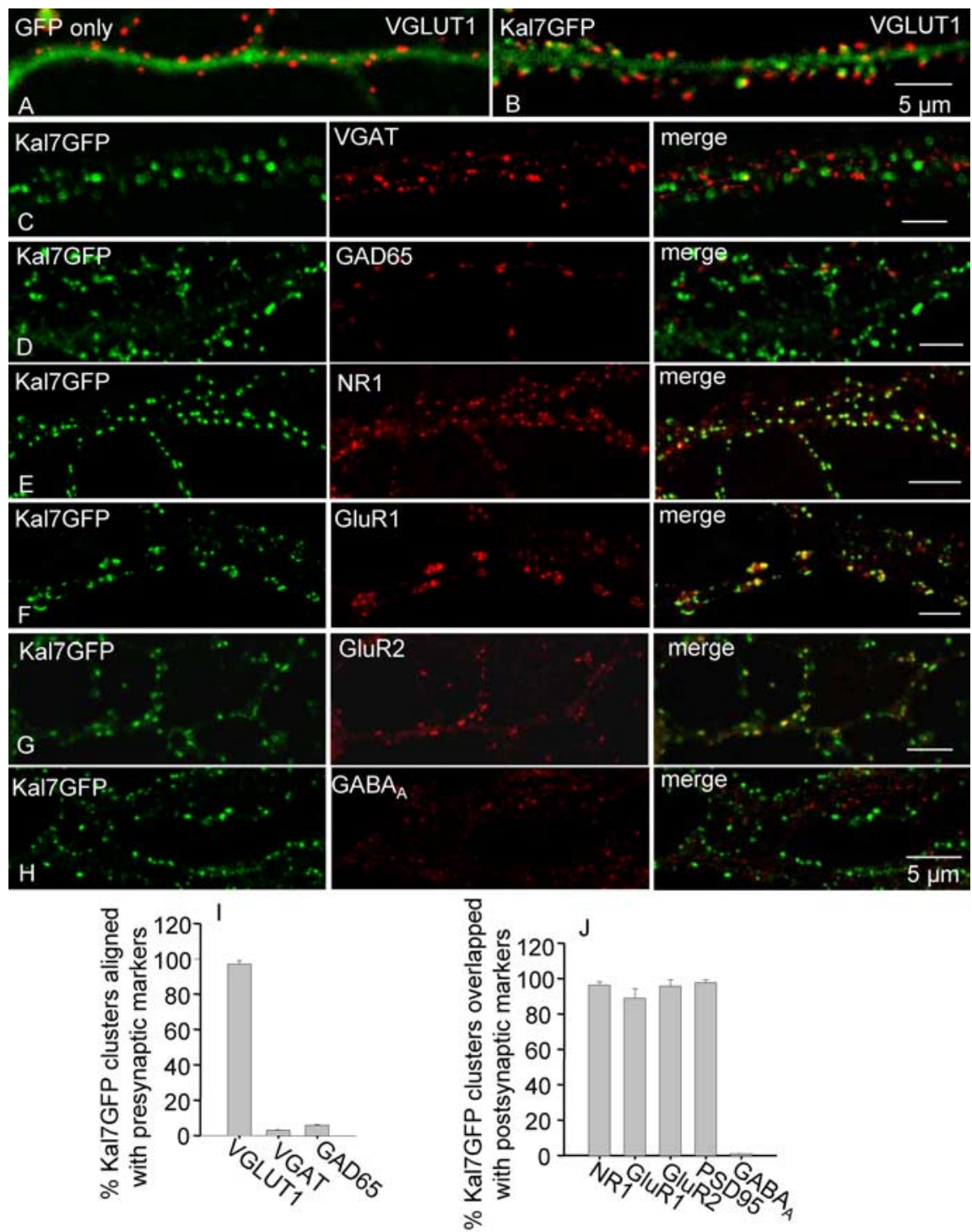

Figure 9. Kal7GFP is localized to the postsynaptic side of excitatory synapses onto interneurons. Cultured hippocampal neurons were transfected with vectors encoding GFP $(\boldsymbol{A})$ or Kal7GFP $(\boldsymbol{B}-\boldsymbol{H})$. At 21 DIV, neurons were subjected to immunostaining with antibody to GFP ( $\boldsymbol{A}-\boldsymbol{H}$, green) and antibodies to VGLUT1, VGAT, NMDA receptor subunit NR1, AMPA receptor subunits GluR1 and GluR2, VGAT, GAD65, or GABA receptor (red). Inhibitory interneurons were identified using antisera to GAD65 (D, red) or GAD65/67 (data not shown). Kal7GFP clusters are closely apposed to markers for excitatory presynaptic endings (I) and coincident with markers for excitatory postsynaptic endings $(J)$ ( $n=14-16$ for each comparison).

\section{Discussion}

Kal7 is localized to excitatory synapses on the dendritic shafts of hippocampal interneurons

Although most glutamatergic synapses in the adult brain occur onto dendritic spines, glutamatergic synapses onto dendritic shafts are common in developing pyramidal neurons and mature GABAergic interneurons (Rao et al., 1998; Gulyas et al., 1999). Both shaft and spine synapses can produce highly localized changes in intracellular calcium (Goldberg et al., 2003). Proteins like PSD-95, along with NMDA and AMPA receptors, are localized to the postsynaptic density of excitatory synapses on the dendritic shafts of interneurons (Allison et al., 1998; Zhang et al., 1999). In mature, dissociated hippocampal GABAergic interneurons, staining for Kal7, PSD-95, and the NR1 subunit of the NMDA receptor is coincident (Fig. 2). Consistent with this, Kal7 clusters align with presynaptic endings identified using antibod- 

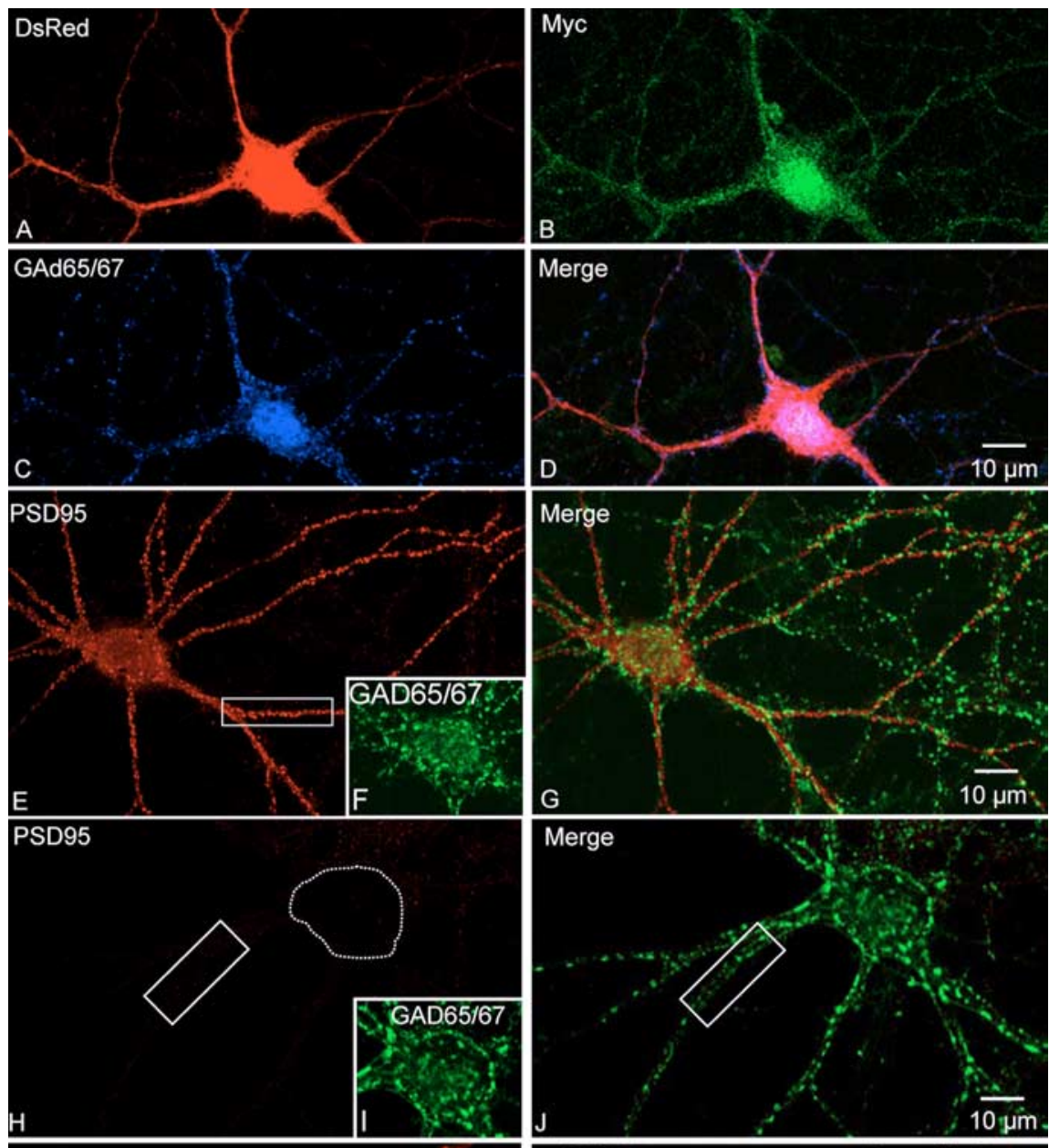

$$
\text { E }
$$
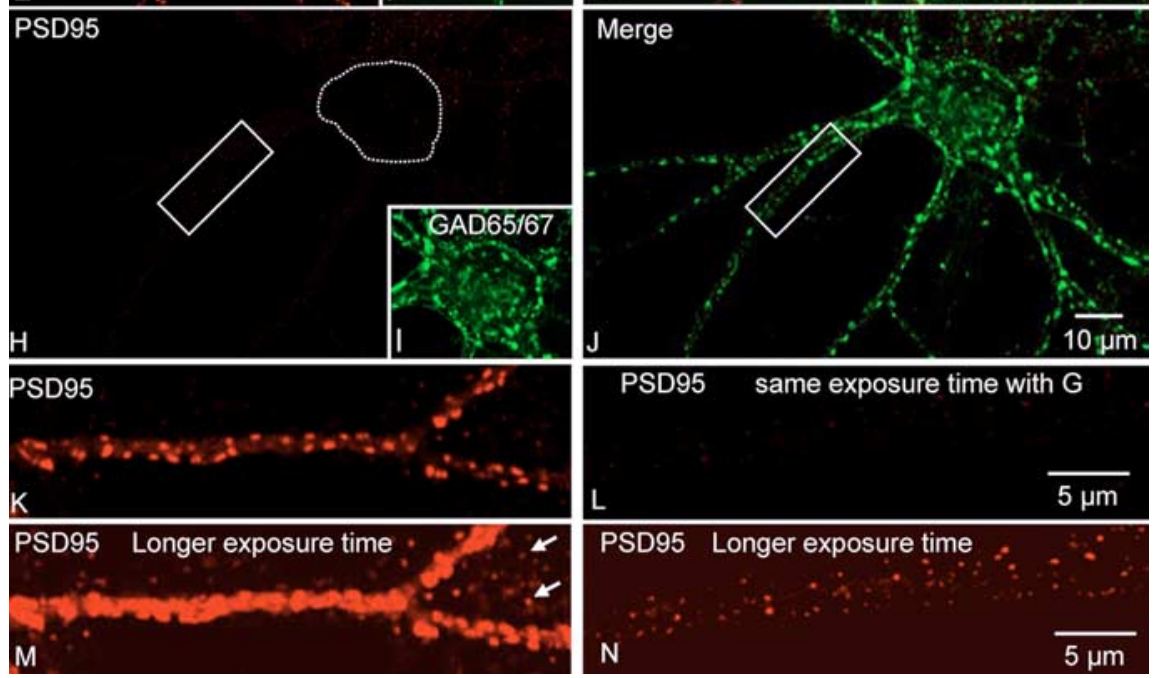

Figure 10. PDZ binding motif is required for Kal7-induced spine formation, but expression of PSD-95 does not mimic expression of Kal7. A-D, Dissociated hippocampal neurons (1 DIV) were transfected simultaneously with vectors encoding DsRed and Kal7 $\Delta$ CT. At P16, neurons were double stained with antibodies to Myc ( $\boldsymbol{B}$, green) and GAD65/67 ( $\boldsymbol{C}$, blue); $\boldsymbol{A}$ is DsRed. $\boldsymbol{D}$ is the merged image. E, Dissociated hippocampal neurons (1 DIV) were transfected with vector encoding PSD-95. Neighboring control neurons in the same dish were not transfected $(\boldsymbol{H})$. Cells were double stained at 18 DIV with antibodies to PSD-95 $(\boldsymbol{E}, \boldsymbol{H}$, red) and GAD65/67 (F, $\boldsymbol{I}$, green). Merged images are shown in $\boldsymbol{G}$ and $\boldsymbol{J}$. $\boldsymbol{K}$ and $\boldsymbol{L}$ are high-power images from boxed dendrites in $\boldsymbol{E}$ and $\boldsymbol{H}$, respectively; longer exposure times $(\boldsymbol{M}, \boldsymbol{N})$ reveal endogenous PSD-95. Arrows in $\boldsymbol{M}$ indicate endogenous PSD-95 staining from the dendrites of a neighboring nontransfected interneuron.

ies to bassoon or VGLUT1. In contrast, Kal7 clusters are not aligned with GAD65-positive terminals.

Kal7 is necessary for maintenance of excitatory synapses onto hippocampal interneurons

To understand the functions of endogenous Kal7 in hippocampal interneurons, levels of Kal7 and $\Delta \mathrm{Kal} 7$ were reduced using a small hairpin RNA and antisense RNA targeted to the $3^{\prime}$-UTR unique to these isoforms. Reducing Kal7 decreased the number of PSD-95 clusters and bassoon-positive presynaptic terminals on the dendrites of interneurons. In this experimental paradigm, levels of Kal12 (and presumably Kal9) were unaltered, identifying
Kal7 as the isoform essential for maintenance of excitatory synapses. Excitatory synapses contain several PDZ domain proteins known to interact with Kal7 in yeast two hybrid assays (PSD-95, Chapsyn-100, SAP102, SAP97, neurabin, spinophilin, afadin, S-SCAM) (Penzes et al., 2001b). By forming an intercellular adhesion complex, postsynaptic neuroligin and presynaptic neurexin serve as a bidirectional trigger for synapse formation, allowing complexes involving PSD-95 to communicate with the presynaptic terminal (Graf et al., 2004; Craig et al., 2006; Dean and Dresbach, 2006). Disruption of this interaction caused by reductions in Kal7 expression could trigger transsynaptic changes that result in elimination of presynaptic inputs.

\section{Overexpression of Kal7 induces the formation of dendritic spines on aspiny interneurons}

As pyramidal neurons develop, synapses shift from dendritic shafts to dendritic spines (Rao et al., 1998; Fiala et al., 1998). Kal7, PSD-95, and spinophilin are known to increase spine formation in neurons that have spines (El Husseini et al., 2000; Feng et al., 2000; Penzes et al., 2001b). However, hippocampal interneurons are spine-free under basal conditions (Benson et al., 1994; Gulyas et al., 1999; Passafaro et al., 2003). Like Kal7, expression of Shank3, a PSD scaffold protein, or GluR2 induced the formation of spine synapses in aspiny neurons that express low levels of endogenous Shank3 or GluR2 in their dendrites (Passafaro et al., 2003; Roussignol et al., 2005). The majority of interneurons express low levels of GluR2 (He et al., 1998), and only $13 \%$ of the endogenous Kal7 clusters contain GluR2. Exogenous Kal7 recruited GluR2 to Kal7-induced spines; experiments using shRNAs specific for Kal7 and GluR2 will be needed to determine whether one gene drives spine formation or there are parallel pathways.

The effects of Kal7 overexpression on spine formation are more dramatic in dissociated neurons than in slice cultures. It is striking that a twofold increase in levels of $\mathrm{Kal} 7$ in dissociated interneurons brings about a $>50$-fold increase in spine density (to 15 spines $/ 10 \mu \mathrm{m}$ ). In slices, a twofold increase in Kal7 levels increases spine density 10 -fold, to three spines $/ 10 \mu \mathrm{m}$. Expression of endogenous Kal7 was not detected until P7 in cultured hippocampal neurons (data not shown). Differences in time of transfection or lack of normal cell-cell interactions may explain the more dramatic response seen in dissociated cell culture.

\section{Overexpression of Kal7 decreases dendritic branching} in interneurons

Dendritic morphology is an important determinant of neuronal information processing. Dendritic growth and branching depend 
on the coordinated action of a number of different extracellular factors (Whitford et al., 2002; Dijkhuizen and Ghosh, 2005). The Rho GTPases are key integrators of factors regulating the dendritic cytoskeleton (Negishi and Katoh, 2005). In slice and dissociated cultures, overexpression of Kal7 caused an increase in the number of dendritic branches within $100 \mu \mathrm{m}$ of the cell soma; in dissociated cells, a decrease in total dendritic length was also observed. These results suggest that Kal7 plays a role in limiting outgrowth of long dendrites in interneurons. Overexpression of the isolated GEF1 domain of Kalirin causes a more dramatic retraction of dendrites in cultured hippocampal interneurons than intact Kal7 (our unpublished observation). Expression of the isolated GEF1 domain also caused dendritic retraction in cortical neurons (Penzes et al., 2001a), indicating that it is a key factor in control of dendritic morphology. Overexpression of PSD-95 caused dendritic retraction in hippocampal neurons (Charych et al., 2006), and the effects of Kal7 on dendritic morphology may involve PSD-95.

Interneurons in the CA1 region of the hippocampus are highly diverse, and different subpopulations have important differences in dendritic morphology (Freund and Buzsaki, 1996; Parra et al., 1998; Maccaferri and Lacaille, 2003). All parvalbumin-expressing CA1 interneurons examined stained for Kal7, with comparable levels in their soma and low levels in their dendrites. CA1 interneurons stained for calbindin or calretinin also contain Kal7 (data not shown). Our quantification focused on the dendrites of interneurons in the CA1 region. Despite the variability inherent in these different subpopulations, the effects of Kal7 on dendritic branching were apparent. As for spine formation, expression of Kal7 in 1 DIV dissociated interneurons, which do not yet express endogenous Kal7, had a more pronounced effect on branching than expression of Kal7 in P9 interneurons in organotypic slices. Coordinate control of the actin cytoskeleton and tubulin is required for normal dendritic growth and maturation, and GEFs that activate Rho GTPases may play a special role in this process (Negishi and Katoh, 2005). As for PSD-95 (El Husseini et al., 2000; Charych et al., 2006), the Kal7 effectors essential to spine formation may differ from the effectors involved in dendritic branching.

\section{Overexpression of Kal7 in hippocampal interneurons induces} formation of excitatory synapses on dendritic spines

The ability of exogenous Kal7 to affect synaptic development was explored in dissociated interneurons. Kal7 interacts directly with PSD-95, and the Kal7 clusters in dendrites overlap completely with PSD-95 clusters; Kal7/PSD-95 coclusters increase and decrease as the level of Kal7 is manipulated. Functional synapses
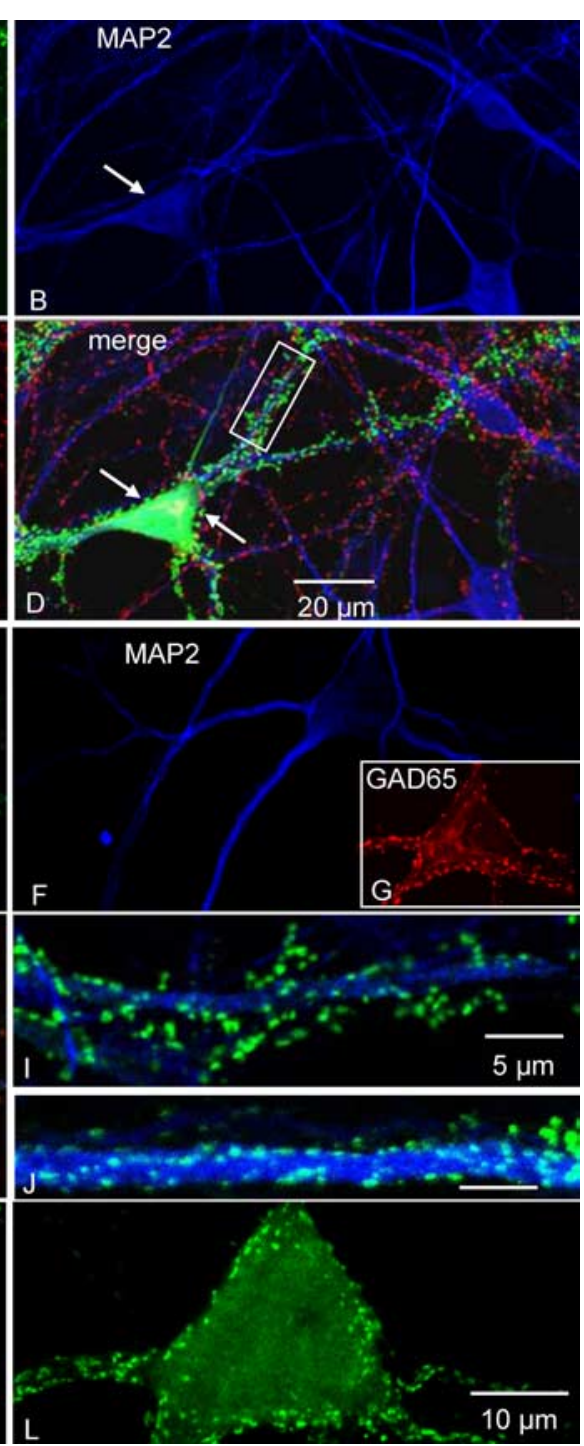

Figure 11. Disruption of Kal7 PDZ binding motif interactions blocks Kal7-induced formation of spine-like structures. Dissociated hippocampal neurons (1 DIV) were transfected with vector encoding Kal7GFP $(\boldsymbol{A}, \boldsymbol{E})$. R7-Kal7CT peptide (10 $\mu$ m; $\boldsymbol{E}-\boldsymbol{H})$ was

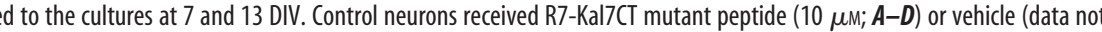
( $\boldsymbol{B}, \boldsymbol{F}$, blue), and GAD65 ( $\boldsymbol{C}, \boldsymbol{G}$, red). $\boldsymbol{I}$ and $\boldsymbol{J}$ are high-power images from boxed dendrites in $\boldsymbol{D}$ and $\boldsymbol{H}$, receptively. $\boldsymbol{K}$ and $\boldsymbol{L}$ are pal interneurons (mutant peptide, $13.2 \pm 1.2 / 10 \mu \mathrm{m}$; Kal7CT peptide, $1.8 \pm 0.9 / 10 \mu \mathrm{m}$; Student's test, $p<0.01 ; n=14$ ). Arrows show spine-like structures in the soma of a GAD65-positive interneuron.

require NMDA and AMPA receptors (Petralia et al., 1999; Liao et al., 2001) and extensive overlap of exogenous Kal7 with endogenous NR1, GluR1, or GluR2 suggests that Kal7-induced synapses are functional. In contrast, exogenous $\mathrm{Kal} 7$ is not colocalized with endogenous $\mathrm{GABA}_{\mathrm{A}}$ receptors.

Bassoon is found in both excitatory and inhibitory endings (Tom et al., 1998). Expression of exogenous Kal7 produces an increase in the number of bassoon-positive structures apposed to the dendrites of interneurons that matches the increase in postsynaptic PSD-95-positive clusters (Fig. 7C,D). The number of inhibitory presynaptic endings onto the dendrites of interneurons is smaller and is not altered by expression of exogenous Kal7. Neither GAD65- nor VGAT-positive endings are apposed to Kal7-positive clusters. Almost all Kal7-positive clusters are apposed to VGLUT1-positive endings. VGLUT1 and 2 localize to distinct excitatory synapses, so the remaining Kal7-positive clus- 
ters may be apposed to VGLUT2-positive endings (Fremeau et al., 2001; Fremeau et al., 2004). We conclude that expression of Kal7 causes formation of dendritic spines that receive glutamatergic inputs. The degree of Kal7-induced spine formation in interneurons is dependent on the level of transfected Kal7 (data not shown).

\section{Kal7-induced spine formation is through interactions between the Kal7 PDZ binding domain and its interactors}

Synaptogenesis is a complex process involving many proteins and two-way communication between presynaptic and postsynaptic elements (Craig et al., 2006). The PDZ domain proteins known to influence spine morphogenesis and synapse development include PSD-95, Shank, spinophilin, NR2, GluR2, Drebin, neuroligin, synCAP, and afadin, and many PDZ proteins localized to excitatory synapses organize glutamate receptors and their associated proteins (Tomita et al., 2001; Kim and Sheng, 2004; Craig et al., 2006). In addition to binding Kal7, PSD-95 binds NMDA receptors and plays a role in their clustering (Kornau et al., 1995; Niethammer et al., 1996). Kal7 lacking a PDZ binding motif does not localize to the postsynaptic density and does not cause spine formation. Although the PDZ binding motif of Kal7 is essential to this response, it is not clear whether known interactors such as PSD-95, spinophilin, neurabin, or afadin (Penzes et al., 2001b) or novel interactors play an essential role in synapse formation.

Disruption of interactions between the Kal7 PDZ binding domain and its interactors by a cell permeant peptide blocks Kal7induced dendritic spine formation. A similar peptide was successfully used to block interaction between PSD-95 and the NMDA receptor, protecting cultured neurons from excitotoxicity (Aarts et al., 2002) and decreasing the number of clusters of PSD-95 and NMDA receptors and their colocalization in the dendrites of dissociated hippocampal neurons (Lim et al., 2003). PDZ proteins localized to the PSD of excitatory synapses control synaptic protein composition and structure (Kim and Sheng, 2004). Many of the PDZ proteins identified in spine synapses are also found at excitatory synapses onto the dendritic shafts of GABAergic interneurons (McBain et al., 1999; Zhang et al., 1999; Mi et al., 2002). Overexpression of PSD-95 in hippocampal interneurons increases the number of GluR1 clusters in dendritic shafts and the frequency of miniature EPSCs (El Husseini et al., 2000). Although overexpression of PSD-95 increased the levels of PSD-95 in interneuron dendritic shafts, it did not cause spine formation. Coexpression of Kal7GFP plus PSD-95 in interneurons did not increase spine density compared with Kal7GFP alone (data not shown), indicating that PSD-95 is not a limiting factor in Kal7-induced spine formation. Overexpression of the GluR2 subunit also induces spine formation in hippocampal interneurons (He et al., 1998; Passafaro et al., 2003). Interneurons clearly have the ability to form spines when instructed to do so, and Kal7 and GluR2 can trigger this response. A crucial next step will be determining whether these factors form part of the same spine-formation pathway in hippocampal interneurons and, if so, identifying the order in which these factors act.

\section{References}

Aarts M, Liu Y, Liu L, Besshoh S, Arundine M, Gurd JW, Wang YT, Salter MW, Tymianski M (2002) Treatment of ischemic brain damage by perturbing NMDA receptor- PSD-95 protein interactions. Science 298:846-850.

Allison DW, Gelfand VI, Spector I, Craig AM (1998) Role of actin in anchoring postsynaptic receptors in cultured hippocampal neurons: differential attachment of NMDA versus AMPA receptors. J Neurosci $18: 2423-2436$.
Anderson TR, Shah PA, Benson DL (2004) Maturation of glutamatergic and GABAergic synapse composition in hippocampal neurons. Neuropharmacology 47:694-705.

Baraban SC, Tallent MK (2004) Interneuron diversity series: interneuronal neuropeptides-endogenous regulators of neuronal excitability. Trends Neurosci 27:135-142.

Benes FM, Berretta S (2001) GABAergic interneurons: implications for understanding schizophrenia and bipolar disorder. Neuropsychopharmacology 25:1-27.

Benson DL, Watkins FH, Steward O, Banker G (1994) Characterization of GABAergic neurons in hippocampal cell cultures. J Neurocytol 23:279-295.

Chang YC, Gottlieb DI (1988) Characterization of the proteins purified with monoclonal antibodies to glutamic acid decarboxylase. J Neurosci 8:2123-2130.

Charych EI, Akum BF, Goldberg JS, Jornsten RJ, Rongo C, Zheng JQ, Firestein BL (2006) Activity-independent regulation of dendrite patterning by postsynaptic density protein PSD-95. J Neurosci 26:10164-10176.

Cobb SR, Halasy K, Vida I, Nyiri G, Tamas G, Buhl EH, Somogyi P (1997) Synaptic effects of identified interneurons innervating both interneurons and pyramidal cells in the rat hippocampus. Neuroscience 79:629-648.

Craig AM, Graf ER, Linhoff MW (2006) How to build a central synapse: clues from cell culture. Trends Neurosci 29:8-20.

Dean C, Dresbach T (2006) Neuroligins and neurexins: linking cell adhesion, synapse formation and cognitive function. Trends Neurosci 29:21-29.

Dijkhuizen PA, Ghosh A (2005) Regulation of dendritic growth by calcium and neurotrophin signaling. Prog Brain Res 147:17-27.

El Husseini AE, Schnell E, Chetkovich DM, Nicoll RA, Bredt DS (2000) PSD-95 involvement in maturation of excitatory synapses. Science 290:1364-1368.

Esclapez M, Tillakaratne NJ, Kaufman DL, Tobin AJ, Houser CR (1994) Comparative localization of two forms of glutamic acid decarboxylase and their mRNAs in rat brain supports the concept of functional differences between the forms. J Neurosci 14:1834-1855.

Feng J, Yan Z, Ferreira A, Tomizawa K, Liauw JA, Zhuo M, Allen PB, Ouimet CC, Greengard P (2000) Spinophilin regulates the formation and function of dendritic spines. Proc Natl Acad Sci USA 97:9287-9292.

Fiala JC, Feinberg M, Popov V, Harris KM (1998) Synaptogenesis via dendritic filopodia in developing hippocampal area CA1. J Neurosci 18:8900-8911.

Fremeau Jr RT, Troyer MD, Pahner I, Nygaard GO, Tran CH, Reimer RJ, Bellocchio EE, Fortin D, Storm-Mathisen J, Edwards RH (2001) The expression of vesicular glutamate transporters defines two classes of excitatory synapse. Neuron 31:247-260.

Fremeau Jr RT, Kam K, Qureshi T, Johnson J, Copenhagen DR, StormMathisen J, Chaudhry FA, Nicoll RA, Edwards RH (2004) Vesicular glutamate transporters 1 and 2 target to functionally distinct synaptic release sites. Science 304:1815-1819.

Freund TF, Buzsaki G (1996) Interneurons of the hippocampus. Hippocampus 6:347-470.

Goldberg JH, Tamas G, Aronov D, Yuste R (2003) Calcium microdomains in aspiny dendrites. Neuron 40:807-821.

Graf ER, Zhang X, Jin SX, Linhoff MW, Craig AM (2004) Neurexins induce differentiation of GABA and glutamate postsynaptic specializations via neuroligins. Cell 119:1013-1026.

Gulyas AI, Toth K, Danos P, Freund TF (1991) Subpopulations of GABAergic neurons containing parvalbumin, calbindin D28k, and cholecystokinin in the rat hippocampus. J Comp Neurol 312:371-378.

Gulyas AI, Megias M, Emri Z, Freund TF (1999) Total number and ratio of excitatory and inhibitory synapses converging onto single interneurons of different types in the CAl area of the rat hippocampus. J Neurosci 19:10082-10097.

He Y, Janssen WG, Vissavajjhala P, Morrison JH (1998) Synaptic distribution of GluR2 in hippocampal GABAergic interneurons and pyramidal cells: a double-label immunogold analysis. Exp Neurol 150:1-13.

Hunt CA, Schenker LJ, Kennedy MB (1996) PSD-95 is associated with the postsynaptic density and not with the presynaptic membrane at forebrain synapses. J Neurosci 16:1380-1388.

Johnson RC, Penzes P, Eipper BA, Mains RE (2000) Isoforms of kalirin, a neuronal Dbl family member, generated through use of different $5^{\prime}$ - and 
3 '-ends along with an internal translational initiation site. J Biol Chem 275:19324-19333.

Kim E, Sheng M (2004) PDZ domain proteins of synapses. Nat Rev Neurosci 5:771-781.

Kornau HC, Schenker LT, Kennedy MB, Seeburg PH (1995) Domain interaction between NMDA receptor subunits and the postsynaptic density protein PSD-95. Science 269:1737-1740.

Liao D, Scannevin RH, Huganir R (2001) Activation of silent synapses by rapid activity-dependent synaptic recruitment of AMPA receptors. J Neurosci 21:6008-6017.

Lim IA, Merrill MA, Chen Y, Hell JW (2003) Disruption of the NMDA receptor-PSD-95 interaction in hippocampal neurons with no obvious physiological short-term effect. Neuropharmacology 45:738-754.

Ma XM, Johnson RC, Mains RE, Eipper BA (2001) Expression of kalirin, a neuronal GDP/GTP exchange factor of the trio family, in the central nervous system of the adult rat. J Comp Neurol 429:388-402.

Ma XM, Mains RE, Eipper BA (2002) Plasticity in hippocampal peptidergic systems induced by repeated electroconvulsive shock. Neuropsychopharmacology 27:55-71.

Ma XM, Huang J, Wang Y, Eipper BA, Mains RE (2003) Kalirin, a multifunctional Rho guanine nucleotide exchange factor, is necessary for maintenance of hippocampal pyramidal neuron dendrites and dendritic spines. J Neurosci 23:10593-10603.

Maccaferri G, Lacaille JC (2003) Interneuron diversity series: hippocampal interneuron classifications-making things as simple as possible, not simpler. Trends Neurosci 26:564-571.

May V, Schiller MR, Eipper BA, Mains RE (2002) Kalirin Dbl-homology guanine nucleotide exchange factor 1 domain initiates new axon outgrowths via RhoG-mediated mechanisms. J Neurosci 22:6980-6990.

McBain CJ, Fisahn A (2001) Interneurons unbound. Nat Rev Neurosci 2:11-23.

McBain CJ, Freund TF, Mody I (1999) Glutamatergic synapses onto hippocampal interneurons: precision timing without lasting plasticity. Trends Neurosci 22:228-235.

McPherson CE, Eipper BA, Mains RE (2002) Genomic organization and differential expression of Kalirin isoforms. Gene 284:41-51.

Mi R, Tang X, Sutter R, Xu D, Worley P, O’Brien RJ (2002) Differing mechanisms for glutamate receptor aggregation on dendritic spines and shafts in cultured hippocampal neurons. J Neurosci 22:7606-7616.

Mody I, Pearce RA (2004) Diversity of inhibitory neurotransmission through GABA(A) receptors. Trends Neurosci 27:569-575.

Nakayama AY, Harms MB, Luo L (2000) Small GTPases Rac and Rho in the maintenance of dendritic spines and branches in hippocampal pyramidal neurons. J Neurosci 20:5329-5338.

Negishi M, Katoh H (2005) Rho family GTPases and dendrite plasticity. Neuroscientist 11:187-191.

Niethammer M, Kim E, Sheng M (1996) Interaction between the C terminus of NMDA receptor subunits and multiple members of the PSD-95 family of membrane-associated guanylate kinases. J Neurosci 16:2157-2163.

Parra P, Gulyas AI, Miles R (1998) How many subtypes of inhibitory cells in the hippocampus? Neuron 20:983-993.

Passafaro M, Nakagawa T, Sala C, Sheng M (2003) Induction of dendritic spines by an extracellular domain of AMPA receptor subunit GluR2. Nature 424:677-681.

Penzes P, Johnson RC, Kambampati V, Mains RE, Eipper BA (2001a) Distinct roles for the two Rho GDP/GTP exchange factor domains of kalirin in regulation of neurite growth and neuronal morphology. J Neurosci 21:8426-8434.

Penzes P, Johnson RC, Sattler R, Zhang X, Huganir RL, Kambampati V, Mains RE, Eipper BA (2001b) The neuronal Rho-GEF Kalirin-7 interacts with PDZ domain-containing proteins and regulates dendritic morphogenesis. Neuron 29:229-242.

Petralia RS, Esteban JA, Wang YX, Partridge JG, Zhao HM, Wenthold RJ, Malinow R (1999) Selective acquisition of AMPA receptors over postnatal development suggests a molecular basis for silent synapses. Nat Neurosci 2:31-36.

Powell EM, Campbell DB, Stanwood GD, Davis C, Noebels JL, Levitt P (2003) Genetic disruption of cortical interneuron development causes region- and GABA cell type-specific deficits, epilepsy, and behavioral dysfunction. J Neurosci 23:622-631.

Ramos B, Baglietto-Vargas D, Rio JC, Moreno-Gonzalez I, Santa-Maria C, Jimenez S, Caballero C, Lopez-Tellez JF, Khan ZU, Ruano D, Gutierrez A, Vitorica J (2006) Early neuropathology of somatostatin/NPY GABAergic cells in the hippocampus of a PS1xAPP transgenic model of Alzheimer's disease. Neurobiol Aging 27:1658-1672.

Rao A, Kim E, Sheng M, Craig AM (1998) Heterogeneity in the molecular composition of excitatory postsynaptic sites during development of hippocampal neurons in culture. J Neurosci 18:1217-1229.

Roussignol G, Ango F, Romorini S, Tu JC, Sala C, Worley PF, Bockaert J, Fagni L (2005) Shank expression is sufficient to induce functional dendritic spine synapses in aspiny neurons. J Neurosci 25:3560-3570.

Ryan XP, Alldritt J, Svenningsson P, Allen PB, Wu GY, Nairn AC, Greengard P (2005) The Rho-specific GEF Lfc interacts with neurabin and spinophilin to regulate dendritic spine morphology. Neuron 47:85-100.

Schiller MR, Blangy A, Huang J, Mains RE, Eipper BA (2005) Induction of lamellipodia by Kalirin does not require its guanine nucleotide exchange factor activity. Exp Cell Res 307:402-417.

Sloviter RS, Nilaver G (1987) Immunocytochemical localization of GABA-, cholecystokinin-, vasoactive intestinal polypeptide-, and somatostatinlike immunoreactivity in the area dentata and hippocampus of the rat. J Comp Neurol 256:42-60.

Tashiro A, Minden A, Yuste R (2000) Regulation of dendritic spine morphology by the rho family of small GTPases: antagonistic roles of Rac and Rho. Cereb Cortex 10:927-938.

Tolias KF, Bikoff JB, Burette A, Paradis S, Harrar D, Tavazoie S, Weinberg RJ, Greenberg ME (2005) The Rac1-GEF Tiam1 couples the NMDA receptor to the activity-dependent development of dendritic arbors and spines. Neuron 45:525-538.

Tom DS, Sanmarti-Vila L, Langnaese K, Richter K, Kindler S, Soyke A, Wex H, Smalla KH, Kampf U, Franzer JT, Stumm M, Garner CC, Gundelfinger ED (1998) Bassoon, a novel zinc-finger CAG/glutamine-repeat protein selectively localized at the active zone of presynaptic nerve terminals. J Cell Biol 142:499-509.

Tomita S, Nicoll RA, Bredt DS (2001) PDZ protein interactions regulating glutamate receptor function and plasticity. J Cell Biol 153:F19-F24.

Wang H, Hu Y, Tsien JZ (2006) Molecular and systems mechanisms of memory consolidation and storage. Prog Neurobiol 79:123-135.

Whitford KL, Dijkhuizen P, Polleux F, Ghosh A (2002) Molecular control of cortical dendrite development. Annu Rev Neurosci 25:127-149.

Zhang W, Vazquez L, Apperson M, Kennedy MB (1999) Citron binds to PSD-95 at glutamatergic synapses on inhibitory neurons in the hippocampus. J Neurosci 19:96-108. 